\title{
Geometrical structure and construction of latin trades
}

\author{
Aleš Drápal* \\ (Communicated by K. Strambach)
}

\begin{abstract}
Latin trades can be interpreted geometrically as oriented surfaces. Those of genus zero are called spherical. The paper contains a construction that shows how all latin trades can be obtained from the spherical ones by a cut-and-paste method that raises the genus.
\end{abstract}

By a latin trade one usually understands a fragment of a latin square for which there exists another fragment that can replace it in the square and has no overlapping cell with the original fragment. In Section 1 we shall introduce an alternative definition that makes it easy to associate with each pair of latin trades a combinatorial oriented surface. In further sections we develop various geometrical properties of these surfaces (sometimes we will call them trading surfaces). We shall show that a trading surface is spherical if and only if each multigon is separating (cf. Section 2 for the definition of the multigon, which is a closed oriented polygonal curve with additional properties). We achieve this result after making a number of auxiliary statements that describe various elementary configurations of surface faces (Sections 3-6). The characterization of trading spherical surfaces by separating multigons is very similar to a well-known characterization of combinatorial spheres as combinatorial surfaces where each polygon is separating. However, the additional structure associated with the notion of a multigon seems to make a direct application of standard theorems difficult.

Once we know that every pair of latin trades that yields a surface of higher genus has to contain a simple non-separating triangle free multigon (Section 7), we can apply a cutand-paste construction (Section 8) that builds all trades of higher genus from the spherical ones (i.e. from the trading spheres, Section 11). The construction can be presented rather simply, but the proof of its correctness is technically quite complicated.

The terminology is standard, with two exceptions. The term "face" is not used in the generic meaning, but will serve as synonymous for "2-cell". Furthermore, we do not

${ }^{*}$ Supported by institutional grant MSM 0021620839. 
insist upon a (combinatorial) surface being connected. Our surfaces can have more than one connected component (but only finitely many).

The ideas presented in this paper can be found already in [7]. However, I did not see clearly the geometrical interpretation of the results at that time, and I felt that the formal setting in which the results were originally formulated is too complicated to warrant publication. After realizing that combinatorial surfaces provide a more intuitive framework, I tried for some time to find shorter proofs that would utilize known facts of combinatorial geometry. This effort failed. On the other hand, results and observations motivated by the geometrical approach to latin bi-trades are increasing [15, 3, 2, 5, 16, 4, 13], further papers are in preparation, and so I finally decided to rewrite and expand [7] in a way that would systematically refer to the interpretation by trading surfaces. Parts of the text were circulated in a preliminary form [9].

\section{Introduction}

By a latin bi-trade we shall mean an ordered pair $\left(T^{\circ}, T^{*}\right)$, where both $T^{\circ}$ and $T^{*}$ are subsets of a set $A_{1} \times A_{2} \times A_{3}$ such that the following conditions hold:

(R1) The sets $T^{\circ}$ and $T^{*}$ are disjoint;

(R2) for all $\left(a_{1}, a_{2}, a_{3}\right) \in T^{\circ}$ and all $r, s \in\{1,2,3\}, r \neq s$, there exists exactly one $\left(b_{1}, b_{2}, b_{3}\right) \in T^{*}$ with $a_{r}=b_{r}$ and $a_{s}=b_{s}$;

(R3) for all $\left(b_{1}, b_{2}, b_{3}\right) \in T^{*}$ and all $r, s \in\{1,2,3\}, r \neq s$, there exists exactly one $\left(a_{1}, a_{2}, a_{3}\right) \in T^{\circ}$ with $a_{r}=b_{r}$ and $a_{s}=b_{s}$.

Lemma 1.1. Suppose that both $\left(a_{1}, a_{2}, a_{3}\right)$ and $\left(a_{1}^{\prime}, a_{2}^{\prime}, a_{3}^{\prime}\right)$ belong to $T^{\circ}\left(\right.$ or $\left.T^{*}\right)$. If they agree on two coordinates, then they agree in the third coordinate as well.

Proof. For example assume that both triples belong to $T^{\circ}$ and agree in the first two coordinates. Consider $\left(a_{1}, a_{2}, b_{3}\right) \in T^{*}$ that is given by (R2). We obtain $a_{3}=a_{3}^{\prime}$ by applying (R3) to $\left(a_{1}, a_{2}, b_{3}\right)$ in place of $\left(b_{1}, b_{2}, b_{3}\right)$.

Both $T^{\circ}$ and $T^{*}$ can thus be regarded as partial latin squares, where $a_{3}$ is the entry value for the cell determined by row $a_{1}$ and column $a_{2}$. This connects our definition of latin bi-trade to the standard definition of latin trade. Latin trades have been studied under various names, and $[14,15,6]$ are excellent sources about the history and origin of the notion. One of the earlier approaches uses the term exchangeable partial groupoid $[11,12]$ which reflects an interpretation of $T^{\circ}$ and $T^{*}$ by partial binary operations, with $a_{1} \circ a_{2}=a_{3}$ for $\left(a_{1}, a_{2}, a_{3}\right) \in T^{\circ}$ and $b_{1} * b_{2}=b_{3}$ for $\left(b_{1}, b_{2}, b_{3}\right) \in T^{*}$.

In addition to (R1)-(R3) we shall always assume in this paper that $\left(T^{\circ}, T^{*}\right)$ satisfies the following conditions:

(R4) The sets $A_{1}, A_{2}$ and $A_{3}$ are pairwise disjoint; and

(R5) for all $\alpha \in A_{1} \cup A_{2} \cup A_{3}$, there exists $\left(a_{1}, a_{2}, a_{3}\right) \in T^{\circ}$ with $a_{i}=\alpha$.

These additional assumptions seem to be reasonable here since we shall investigate the intrinsic structure of latin trades, and not their embedding into latin squares. However, when representing a latin bi-trade by tables, the condition (R4) is often violated, as in 
the example below. This section explains how to derive three permutations of certain properties from each latin bi-trade. This process will transform our example into Figure 2. The rows correspond to cycles in the figure that are labelled $\alpha_{i}, 1 \leq i \leq 4$, columns to cycles $\beta_{j}$ and symbols (the set $A_{3}$ ) to cycles $\gamma_{k}$. The table on the right indicates how the points in the figure are connected to the cells of the trade.

\begin{tabular}{|c|c|c|c|c|c|c|c|c|c|c|c|c|c|c|c|c|c|c|c|c|}
\hline$\circ$ & 1 & 2 & 3 & 4 & 5 & 6 & $*$ & 1 & 2 & 3 & 4 & 5 & 6 & & 1 & 2 & 3 & 4 & 5 & 6 \\
\hline 1 & & 4 & & 2 & & 5 & 1 & & 2 & & 5 & & 4 & 1 & & $h$ & & $i$ & & $b$ \\
\hline 2 & 4 & 2 & 3 & 1 & & & 2 & 3 & 4 & 1 & 2 & & & 2 & $g$ & $m$ & $n$ & $j$ & & \\
\hline 3 & 3 & & 1 & & 6 & & 3 & 6 & & 3 & & 1 & & 3 & $l$ & & $k$ & & $e$ & \\
\hline 4 & 6 & & & 5 & 1 & 4 & 4 & 4 & & & 1 & 6 & $J$ & 4 & $f$ & & & $c$ & $a$ & $a$ \\
\hline
\end{tabular}

Latin bi-trades $\left(T^{\circ}, T^{*}\right), T^{\circ} \subseteq A_{1} \times A_{2} \times A_{3}$, and $\left(S^{\circ}, S^{*}\right), S^{\circ} \subseteq B_{1} \times B_{2} \times B_{3}$, are said to be isotopic if there exist bijections $\varphi_{i}: A_{i} \rightarrow B_{i}, 1 \leq i \leq 3$, such that $\left(a_{1}, a_{2}, a_{3}\right) \in T^{\circ}$ (or $\left.T^{*}\right)$ if and only if $\left(\varphi_{1}\left(a_{1}\right), \varphi_{2}\left(a_{2}\right), \varphi_{3}\left(a_{3}\right)\right) \in S^{\circ}$ (or $\left.S^{*}\right)$.

Consider $r, s \in\{1,2,3\}, r \neq s$, and denote by $t$ the third element of $\{1,2,3\}$. A permutation $\sigma_{s, r}$ of $T^{\circ}$ is defined in two stages as follows:

(1) For $a=\left(a_{1}, a_{2}, a_{3}\right) \in T^{\circ}$ find $b=\left(b_{1}, b_{2}, b_{3}\right) \in T^{*}$ with $a_{s}=b_{s}$ and $a_{t}=b_{t}$;

(2) put $\sigma_{s, r}(a)=\left(a_{1}^{\prime}, a_{2}^{\prime}, a_{3}^{\prime}\right) \in T^{\circ}$, where $a_{r}^{\prime}=b_{r}$ and $a_{t}^{\prime}=b_{t}$.

Thus $\sigma_{s, r}$ changes the $r$-th coordinate first, and then the $s$-th coordinate. Clearly $a_{t}^{\prime}=a_{t}, a_{s}^{\prime} \neq a_{s}$ and $a_{r}^{\prime} \neq a_{r}$. Stage (1) describes a bijection $\beta_{r}: T^{\circ} \rightarrow T^{*}$ and stage (2) defines the inverse bijection $\beta_{s}^{-1}: T^{*} \rightarrow T^{\circ}$. The equality $\sigma_{s, r}=\beta_{s}^{-1} \beta_{r}$ implies the next lemma in an obvious way.

Lemma 1.2. Let $r, s, t \in\{1,2,3\}$ be pairwise distinct. Then $\sigma_{t, s} \sigma_{s, r}=\sigma_{t, r}$ and $\sigma_{r, s}=$ $\sigma_{s, r}^{-1}$.

From Lemma 1.2 it follows that using two subscripts in $\sigma_{r, s}$ is superfluous. Instead we shall use the notation $\tau_{1}=\sigma_{2,3}, \tau_{2}=\sigma_{3,1}$ and $\tau_{3}=\sigma_{1,2}$. These three permutations will sometimes be called the structural permutations of $\left(T^{\circ}, T^{*}\right)$, and $\left(\tau_{1}, \tau_{2}, \tau_{3}\right)$ will be known as its structural triple. Lemma 1.2 gives

Corollary 1.3. Let $\left(T^{\circ}, T^{*}\right)$ be a latin bi-trade. Then $\tau_{1} \tau_{2} \tau_{3}=\tau_{2} \tau_{3} \tau_{1}=\tau_{3} \tau_{1} \tau_{2}$ is the identity on $T^{\circ}$.

Each permutation $\tau_{i}$ decomposes into cycles, and the number of such cycles will be known as the permutational order of the latin bi-trade. We shall call it just order if there is no danger of misunderstanding. The number of elements of $T^{\circ}$ (which is identical with the number of elements of $T^{*}$ ) will be called the size of the latin bi-trade.

We see that the triple $\left(\tau_{1}, \tau_{2}, \tau_{3}\right)$ fulfils the following two conditions:

(P1) A cycle of $\tau_{r}$ has at most one point in common with a cycle of $\tau_{s}$, whenever $1 \leq$ $r<s \leq 3$; and

(P2) all permutations $\tau_{i}, 1 \leq i \leq 3$, are fixed point free and $\tau_{1} \tau_{2} \tau_{3}$ is the identity.

Let $\tau$ be a permutation of a set $X$ and let $\rho=\left(x_{1} x_{2} \ldots x_{k}\right)$ be one of its cycles (with respect to the cyclic decomposition of $\tau$ ). A point $x \in X$ is said to be incident to 
$\rho$ if and only if $x=x_{j}$ for some $j, 1 \leq j \leq k$. Thus $x$ is incident to $\rho$ if and only if $\rho$ moves $x$.

Let $\left(T^{\circ}, T^{*}\right)$ be a latin bi-trade with structural triple $\left(\tau_{1}, \tau_{2}, \tau_{3}\right)$. Consider $i \in\{1,2,3\}$ and $a=\left(a_{1}, a_{2}, a_{3}\right) \in T^{\circ}$, and denote by $\rho$ the cycle of $\tau_{i}$ that moves $a$. The coordinate $a_{i}$ does not change under $\tau_{i}$, and so it is an invariant of $\rho$. If for every $i \in\{1,2,3\}$ and every $a_{i} \in A_{i}$ there exists only one cycle $\rho$ of $\tau_{i}$ with the invariant $a_{i}$, then $\left(T^{\circ}, T^{*}\right)$ is said to be a separated latin bi-trade.

Proposition 1.4. Let $\tau_{i}, 1 \leq i \leq 3$, be permutations of a set $X$ that satisfy (P1) and (P2). Denote by $A_{i}, 1 \leq i \leq 3$, the set of all cycles of the permutation $\tau_{i}$. Define $T^{\circ}, T^{*} \subseteq A_{1} \times A_{2} \times A_{3}$ by

$$
\begin{aligned}
\left(\rho_{1}, \rho_{2}, \rho_{3}\right) \in T^{\circ} \Longleftrightarrow & \text { all } \rho_{i}, 1 \leq i \leq 3 \text {, are incident to some } x \in X, \quad \text { and } \\
\left(\rho_{1}, \rho_{2}, \rho_{3}\right) \in T^{*} \Longleftrightarrow & \text { there exists an element } x \in X \text { such that } \rho_{3} \text { moves } x, \\
& \rho_{2} \text { moves } \rho_{3}(x), \text { and } \rho_{1} \text { moves } \rho_{2}\left(\rho_{3}(x)\right) .
\end{aligned}
$$

Then $\left(T^{\circ}, T^{*}\right)$ is a latin bi-trade.

Proof. Let $\left(\rho_{1}, \rho_{2}, \rho_{3}\right) \in T^{*}$ be determined by $x \in X$. Set $y=\rho_{3}(x)$ and observe that $\rho_{1} \rho_{2}(y)=\tau_{1} \tau_{2} \tau_{3}(x)=x$. Thus $\rho_{2}$ moves $y, \rho_{1}$ moves $\rho_{2}(y)$, and $\rho_{3}$ moves $\rho_{1} \rho_{2}(y)$. The definition of $T^{*}$ in the proposition is hence invariant with respect to the cyclic shift of indices, and so to prove (R2) and (R3) we can assume, say, $r=1$ and $s=3$.

Note that (R1) follows from the fact that all $\tau_{i}$ are fixed point free.

If $\left(\rho_{1}, \rho_{2}, \rho_{3}\right) \in T^{*}$, then by the definition of $T^{*}$ there exists $x \in X$ which is incident both to $\rho_{3}$ and $\rho_{1}$. Such an $x$ is unique by (P1), and hence there exists a unique $\rho_{2}^{\prime}$ with $\left(\rho_{1}, \rho_{2}^{\prime}, \rho_{3}\right) \in T^{\circ}$ (this $\rho_{2}^{\prime}$ is the cycle of $\tau_{2}$ that moves $x$ ). We have proved (R3).

To prove (R2) consider $\left(\rho_{1}, \rho_{2}, \rho_{3}\right) \in T^{\circ}$, and let $x$ be the common point of $\rho_{i}, 1 \leq$ $i \leq 3$. By (P1), $\tau_{1} \tau_{2} \rho_{3}(x)$ equals $x$. Since $\rho_{1}$ is the cycle of $\tau_{1}$ which moves $x$, we have, in fact, $\rho_{1} \tau_{2} \rho_{3}(x)=x$. Denote by $\rho_{2}^{\prime}$ the cycle of $\tau_{2}$ that moves $\rho_{3}(x)$. We obtain $\left(\rho_{1}, \rho_{2}^{\prime}, \rho_{3}\right) \in T^{*}$. The cycle $\rho_{2}^{\prime}$ is determined uniquely since any such $\rho_{2}^{\prime}$ has to move some $\rho_{3}(y)$ by the definition of $T^{*}$, where $y$ is incident to both $\rho_{1}$ and $\rho_{3}$. However, such a $y$ has to equal $x$, by (P1) and by the preceding part of the proof.

Starting from a latin bi-trade $S=\left(S^{\circ}, S^{*}\right), S^{\circ} \subseteq B_{1} \times B_{2} \times B_{3}$, one can first consider its structural triple $\left(\tau_{1}, \tau_{2}, \tau_{3}\right)$, and then construct the latin bi-trade $T=\left(T^{\circ}, T^{*}\right)$ by means of Proposition 1.4. The structure of $T$ closely follows the structure of $S$. For example, if $a_{i}$ is the invariant of $\rho_{i}, 1 \leq i \leq 3$, then $\left(\rho_{1}, \rho_{2}, \rho_{3}\right) \in T^{\circ}$ implies $\left(a_{1}, a_{2}, a_{3}\right) \in S^{\circ}$, and $\left(\rho_{1}, \rho_{2}, \rho_{3}\right) \in T^{*}$ implies $\left(a_{1}, a_{2}, a_{3}\right) \in S^{*}$. One can say that each $a_{i} \in B_{i}$ is divided into so many elements of $A_{i}$, however many cycles $\rho$ of $\tau_{i}$ there are that have $a_{i}$ as their invariant. This means that if $S$ is separated, then there is a natural bijection between $B_{i}$ and $A_{i}$, and these bijections yield an isotopism. In fact, $T$ is isotopic to $S$ if and only if $S$ is separated. These facts are natural and easy. A formal proof appears in [10], together with a formal proof of the (more or less obvious) fact that the construction of Proposition 1.4 always yields a separated latin bi-trade.

The structure of latin bi-trades is determined by the structure of separated latin bitrades and the study of separated latin bi-trades is equivalent to the study of permutation 
triples $\left(\tau_{1}, \tau_{2}, \tau_{3}\right)$ that fulfil (P1) and (P2). These two properties also suffice to describe the connection to (combinatorial) oriented surfaces. We shall obtain such surfaces in a discrete manner from appropriate sets of points, edges and faces.

The set of points, for a given triple $\left(\tau_{1}, \tau_{2}, \tau_{3}\right)$, coincides with the set $X$ upon which the permutations $\tau_{i}$ act. The edges are the sets $\left\{x, \tau_{i}(x)\right\}, x \in X$ and $i \in\{1,2,3\}$. Such an edge appears with multiplicity 2 when $\tau_{i}^{2}(x)=x$. An edge can have positive orientation $\left(x, \tau_{i}(x)\right)$ and negative orientation $\left(\tau_{i}(x), x\right)$. In this paper we shall call 2cells faces, and by a face we shall always understand a 2-cell. The faces fall into two categories: cyclic faces and triangular faces. Every cyclic face $\left(x_{1}, x_{2}, \ldots, x_{k}\right)$ expresses a cycle of $\tau_{i}, 1 \leq i \leq 3$. Thus $\tau_{i}\left(x_{j}\right)=x_{j+1}, 1 \leq j<k$ and $\tau_{i}\left(x_{k}\right)=x_{1}$. All triangular faces are of the form $\left(x, \tau_{2} \tau_{3}(x), \tau_{3}(x)\right), x \in X$. (Equivalent expressions are $\left(x, \tau_{1} \tau_{2}(x), \tau_{2}(x)\right),\left(\tau_{1}(x), x, \tau_{2}^{-1}(x)\right)$, etc.) Note that the given presentation of cyclic faces orders vertices by positive orientation of edges, while negative orientation is used for the triangular faces. Each $x \in X$ appears in exactly three different cyclic faces:

$$
\left(\ldots, \tau_{3}^{-1}(x), x, \tau_{3}(x), \ldots\right),\left(\ldots, \tau_{2}^{-1}(x), x, \tau_{2}(x), \ldots\right) \text { and }\left(\ldots, \tau_{1}^{-1}(x), x, \tau_{1}(x), \ldots\right) \text {, }
$$

and in exactly three different triangular faces:

$$
\left(\tau_{2}(x), x, \tau_{3}^{-1}(x)\right), \quad\left(\tau_{1}(x), x, \tau_{2}^{-1}(x)\right) \quad \text { and } \quad\left(\tau_{3}(x), x, \tau_{1}^{-1}(x)\right) .
$$

By interspersing cyclic and triangular faces we clearly get a cyclic ordering of all faces that include $x$ (see Figure 1). That induces a cyclic ordering of all edges containing $x$ such that each edge is contained in exactly two faces, and has opposite orientation in each of these two adjacent faces. The existence of such an ordering means that we have obtained a combinatorial surface. The surface is oriented since one obtains a coherent orientation when the triangular faces are oriented in a positive way.

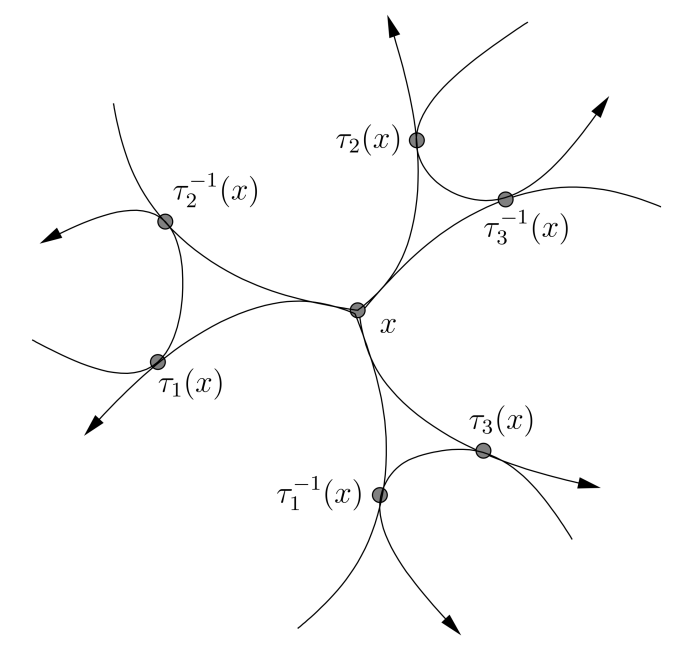

Figure 1. Triangular and cyclic faces incident to a point $x$. 
The connection between latin trades and oriented surfaces was first published in [8]. See also [15] for a detailed exposition with examples.

Let $T=\left(T^{\circ}, T^{*}\right)$ be a latin bi-trade. Elements $a=\left(a_{1}, a_{2}, a_{3}\right) \in T^{\circ}$ and $b=$ $\left(b_{1}, b_{2}, b_{3}\right) \in T^{\circ}$ are said to be connected if $a_{i}=b_{i}$ for some $i \in\{1,2,3\}$ (we also say that $a$ and $b$ are connected by $a_{i}$ ). The latin bi-trade $T$ is said to be connected if for all $x, y \in T^{\circ}$ there exists a sequence $x=x_{0}, x_{1}, \ldots, x_{k}=y$ such that $x_{j}$ and $x_{j+1}$ are connected for every $j, 1 \leq j \leq k$. If $T$ is separated, then one can easily see that $T$ is connected if and only if the oriented surface of $T$ is connected. Furthermore, in a separated latin bi-trade one has that $a$ and $b$ are connected if and only if there exists a cycle that moves both $a$ and $b$. A trading surface $\left(\tau_{1}, \tau_{2}, \tau_{3}\right)$ is thus connected if and only if the permutation group $\left\langle\tau_{1}, \tau_{2}, \tau_{3}\right\rangle$ is transitive.

Proposition 1.5. Let $T=\left(T^{\circ}, T^{*}\right)$ be a connected separated latin bi-trade. Then

$$
2 \operatorname{genus}(T)+\operatorname{order}(T)=\operatorname{size}(T)+2 .
$$

Proof. The oriented surface of $T$ has $f=\operatorname{order}(T)+\operatorname{size}(T)$ faces, $e=3 \operatorname{size}(T)$ edges and $p=\operatorname{size}(T)$ points. The equality of the proposition thus expresses the Euler identity $2(1-g)+e=f+p$, where $g$ is the genus of the surface.

Call a latin bi-trade $T$ spherical if its oriented surface is a combinatorial sphere (i.e., the surface is connected and its genus is equal to 0; Figure 2 gives an example). In

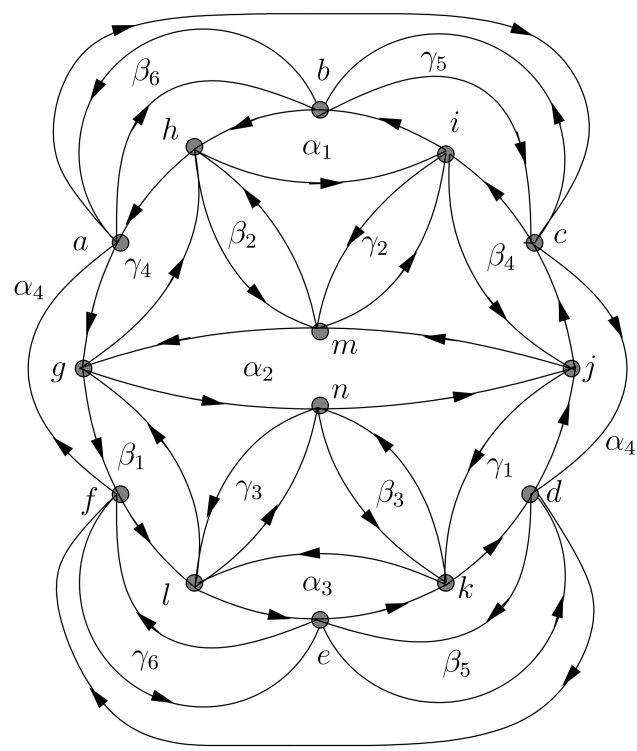

Figure 2. A spherical latin bi-trade of order 16 and size 14. The cyclic faces are labelled by Greek letters, the other faces are triangular. Note that all outer edges belong to the cycle $\alpha_{4}$. 
such a latin bi-trade order $(T)>\operatorname{size}(T)$. If $T$ is spherical and separated, then $T^{\circ} \subseteq$ $A_{1} \times A_{2} \times A_{3}$ implies that $\left|A_{i}\right|>\operatorname{size}(T) / 3$ for some $i \in\{1,2,3\}$. The size of $T$ equals the sum of cycle lengths computed over all cycles of $\tau_{i}$. There are $\left|A_{i}\right|$ such cycles, and the latter inequality could not hold, if each cycle were of size at least three. We have proved

Lemma 1.6. Let $\left(T^{\circ}, T^{*}\right)$ be a spherical latin bi-trade and let $\left(\tau_{1}, \tau_{2}, \tau_{3}\right)$ be its structural triple. Then there exists $x \in T^{\circ}$ and $i \in\{1,2,3\}$ such that $\tau_{i}^{2}(x)=x$.

Lemma 1.6 can be utilized to show the existence of a reduction/generation scheme that yields all spherical latin bi-trades from those of size 4. This is described in [10]. This paper is concerned with a reduction/generation scheme for connected non-spherical separated latin bi-trades. The basic idea is to consider every positively oriented closed non-intersecting path upon the surface of $T$ which never travels twice along the same cyclic face (this will be called a multigon), and to find one with certain additional properties, one of which states that the path does not separate the surface into two components. The surface is then cut along such a path, and two planar substructures are pasted. This simple and standard idea needs quite a lot of technical auxiliary notions, and their precision and clarification is more or less the content of the rest of this paper.

Each latin bi-trade $T=\left(T^{\circ}, T^{*}\right)$ yields the dual latin bi-trade $\tilde{T}=\left(T^{*}, T^{\circ}\right)$. The structural permutations of $\tilde{T}$ move elements of $T^{*}$ and will be denoted by $\tilde{\tau}_{i}, 1 \leq i \leq 3$. It is easy to see that $T$ is separated (or connected) if and only if $\tilde{T}$ is separated (or connected).

\section{Closed oriented polygonal curves}

Let $\tau_{i}, 1 \leq i \leq 3$, be permutations of a set $X$ such that the triple $\left(\tau_{1}, \tau_{2}, \tau_{3}\right)$ satisfies conditions (P1) and (P2). Use Proposition 1.4 to construct a latin bi-trade $\left(T^{\circ}, T^{*}\right)$, and identify $T^{\circ}$ with $X$. The oriented combinatorial surface associated with $\left(T^{\circ}, T^{*}\right)$ will be denoted by $S$.

By an oriented edge of $S$ we shall mean an edge in its positive orientation, i.e. a pair $\left(x, \tau_{i}(x)\right)$, where $x \in X$ and $i \in\{1,2,3\}$. The set of all cycles of $\tau_{i}$ will be denoted by $A_{i}$, as in Proposition 1.4.

A sequence $x_{1} \ldots x_{k}$ of elements of $X$ is called a closed oriented polygonal curve if for each $j, 1 \leq j \leq k$, there exists $\sigma_{j} \in \bigcup A_{i}$ such that $\sigma_{j}\left(x_{j}\right)=x_{j+1}$ (set $x_{k+1}=x_{1}$ and $x_{0}=x_{k}$; similar conventions will be used elsewhere as well). Pairs $\left(x_{j}, x_{j+1}\right)$ will be called the (oriented) edges of the polygonal curve.

Consider the sequence $j_{1}<\cdots<j_{r}$ consisting of all $j, 1 \leq j \leq k$, such that $\sigma_{j} \neq \sigma_{j-1}$. The cyclic sequence $\left(\sigma_{j_{1}}, \ldots, \sigma_{j_{r}}\right)$ will be called the profile of the curve, and the points $x_{j_{1}}, \ldots, x_{j_{r}}$ are called the vertices of the curve. Every $x_{j}, 1 \leq j \leq k$, is a point of the curve, and so we have vertex points and non-vertex points. It is clear that a curve has no vertex points if and only if its profile is empty, and this takes place if and only if all edges of the curve are induced by a single cycle.

Let $C$ be a closed oriented polygonal curve with profile $\left(\sigma_{1}, \ldots, \sigma_{r}\right)$ and vertices $\left(v_{1}, \ldots, v_{r}\right)$. We shall always keep the convention that $\sigma_{j}$ and $\sigma_{j+1}$ meet in the vertex 
$v_{j+1}$. Call $C$ a multigon if (1) its profile is nonempty, and (2) $\sigma_{i} \neq \sigma_{j}$ and $v_{i} \neq v_{j}$, whenever $1 \leq i<j \leq r$. Call the multigon $C$ simple if $\sigma_{i}$ meets $\sigma_{j}, i \neq j$, if and only if $i=j \pm 1$.

Turn to Figure 2 for an example. The sequence gnlekdjcibha is a closed oriented polygonal curve with profile $\left(\alpha_{2}, \gamma_{3}, \alpha_{3}, \gamma_{1}, \beta_{4}, \alpha_{1}, \gamma_{4}\right)$ and with vertices $g, n, l, k, j, i$ and $h$. We see that the curve is a multigon, but this multigon is not simple as $\alpha_{2}$ and $\gamma_{1}$ meet in $j$. The opposite multigon (see below) is equal to ghijklnjm. This multigon contains $j$ once as a vertex and once as a non-vertex point.

In combinatorial topology one usually calls a polygonal curve $x_{1} \ldots x_{k}$ simple if $x_{i} \neq x_{j}$ when $1 \leq i<j \leq k$. One of the basic theorems states that a connected combinatorial surface is a combinatorial sphere if and only if each simple closed polygonal curve is separating (e.g., see [1, Theorem 7.7]). In Section 7 we shall obtain a similar characterization of spherical latin bi-trades by means of separating multigons. To get the result we will have to overcome certain technical difficulties that arise from the fact that the multigons in the sense above need not be simple (a vertex $v_{j}=x_{i}$ can appear also as $x_{i^{\prime}}, i^{\prime} \neq i$, on a non-vertex position, and be moved by some $\sigma_{j^{\prime}}, j^{\prime} \notin\{j-1, j\}$. A nonvertex point can be equal to $x_{i}$ for up to three indices $i$, and be moved by up to three cycles $\sigma_{j}$.) Other technical problems have to do with the fact that multigons in the sense of our definition are oriented curves, and so the amount of curves at our disposal is smaller when we wish to show that a latin bi-trade of positive genus yields a non-separating multigon.

Let us now, for a while, consider a connected combinatorial surface $K$ without multiple edges that need not have any connection to latin bi-trades. Let $C$ be a closed polygonal curve in $K$. What does it mean that $C$ is separating? This is clear topologically: the surface becomes disconnected when $C$ is removed. However, here we shall need a combinatorial definition. We shall define (combinatorial) components of $K$ that are induced by $C$ in such a way that there will be at most two components, even in the case when $C$ has repeated points.

Recall that the star of the point $x$ is formed by the set of all faces that contain $x$. Any two edges $\left\{y_{1}, x\right\}$ and $\left\{y_{2}, x\right\}, y_{1} \neq y_{2}$, divide the star of $x$ into two connected components. We shall call them components induced by $\left(y_{1}, x, y_{2}\right)$.

Let $C$ be equal to $x_{1} \ldots x_{k}$. Furthermore, let $E_{0}$ be a face with the edge $\left\{x_{k}, x_{1}\right\}$, and let $F_{0}$ be the other face containing $\left\{x_{k}, x_{1}\right\}$. For each $j, 1 \leq j \leq k$, construct faces $E_{j}$ and $F_{j}$ containing $\left\{x_{j}, x_{j+1}\right\}$ in such a way that $E_{j-1}$ and $E_{j}$ are in the same component induced by $\left(x_{j-1}, x_{j}, x_{j+1}\right)$. (Faces $F_{j-1}$ and $F_{j}$ are then in the other component; the indices are taken modulo $k$.)

Let $\sim_{C}$ be the smallest equivalence relation on the set of all faces of $K$ such that

(1) $E_{j-1} \sim_{C} E_{j}$ and $F_{j-1} \sim_{C} F_{j}$ for every $j, 1 \leq j \leq k$; and

(2) $E \sim_{C} F$ whenever $E$ and $F$ share an edge distinct from $\left\{x_{j}, x_{j+1}\right\}, 1 \leq j \leq k$.

Call the curve $C$ separating if the equivalence relation $\sim_{C}$ has more than one equivalence class.

To see that $\sim_{C}$ can have at most two equivalence classes, consider the smallest equivalence relation $\approx$ that contains $\sim_{C}$, and satisfies $E_{0} \approx F_{0}$. We shall show that $\approx$ has only one equivalence class. For that it suffices to prove $E_{j} \approx F_{j}$ for every $j, 1 \leq j \leq k$, 
since $K$ is assumed to be a connected surface. However, that follows by induction, as $E_{j} \sim_{C} E_{j-1} \approx F_{j-1} \sim_{C} F_{j}$.

If $E_{0}=F_{k}$, then $C$ is never separating. If $E_{0}=E_{k}$, then also $F_{0}=F_{k}$. In such a case we shall call $\left(E_{1}, \ldots, E_{k}\right)$ and $\left(F_{1}, \ldots, F_{k}\right)$ the border sequences of $C$.

In the definitions above we needed to assume that $K$ is without multiple edges in order to have an edge $\{x, y\}$ uniquely determined by the points $x$ and $y$. Let us now return to the combinatorial surface $S$ derived from $\left(\tau_{1}, \tau_{2}, \tau_{3}\right)$. For any $x, y \in X$ there exists at most one oriented edge $(x, y)$. Hence the above definitions can be applied to any closed polygonal oriented curve $C$ of $S$. In particular, we can speak of separating and non-separating multigons.

Every cyclic face gives rise to a simple closed oriented polygonal curve with empty profile. Every triangular face can be uniquely represented as $\left(x_{1}, x_{2}, x_{3}\right)$, where $x_{i}$ is moved by $\sigma_{i} \in A_{i}$ to $x_{i-1}$, and $\left(\sigma_{1}, \sigma_{2}, \sigma_{3}\right) \in T^{*}$. By moving around a triangular face along oriented edges one gets a multigon $x_{3} x_{2} x_{1}$ with profile $\left(\sigma_{3}, \sigma_{2}, \sigma_{1}\right)$ and vertices $\left(x_{3}, x_{2}, x_{1}\right)$. Such multigons will be called triangles.

A triangle is clearly a separating multigon. Our aim is to show that $S$ is a sphere if and only if it is connected and every multigon is separating.

If $i, i^{\prime} \in\{1,2,3\}$, then there exists a unique $\varepsilon \in\{-1,0,1\}$ with $i^{\prime}-i \equiv \varepsilon \bmod 3$. For $\sigma^{\prime} \in A_{i^{\prime}}$ and $\sigma \in A_{i} \operatorname{set} \operatorname{sgn}\left(\sigma^{\prime}, \sigma\right)=\varepsilon$ if $\sigma^{\prime}$ and $\sigma$ have a common point, and set $\operatorname{sgn}\left(\sigma^{\prime}, \sigma\right)=0$ if they have no common point.

Let $C$ be the closed polygonal curve $x_{1} \ldots x_{k}$, and let $\sigma_{j} \in \bigcup A_{i}$ be the cycle that moves $x_{j}$ to $x_{j+1}$. For every $j, 1 \leq j \leq k$, put $\operatorname{sgn}_{C}\left(x_{j}\right)=\operatorname{sgn}\left(\sigma_{j-1}, \sigma_{j}\right)$. Note that if $C$ is a multigon, then $x_{j}$ is a vertex if and only if $\operatorname{sgn}_{C}\left(x_{j}\right)=\varepsilon \neq 0$. Call the vertex acute if $\varepsilon=1$, and obtuse if $\varepsilon=-1$. Set $\operatorname{sgn}_{C}(x)=0$ also for the points of $S$ that are not on $C$.

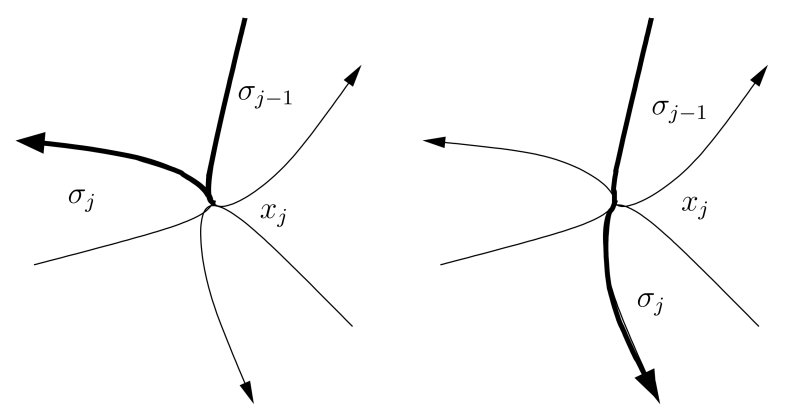

Figure 3. Put $\varepsilon=\operatorname{sgn}\left(\sigma_{j-1}, \sigma_{j}\right)$. The vertex $x_{j}$ is acute if $\varepsilon=1$ (on the left) and obtuse if $\varepsilon=-1$ (on the right).

Lemma 2.1. The sum $\sum \operatorname{sgn}_{C} x_{j}, 1 \leq j \leq k$, is divisible by 3 for every oriented closed polygonal curve $C$.

Proof. Set $s_{j}=\operatorname{sgn}_{C}\left(x_{1}\right)+\cdots+\operatorname{sgn}_{C}\left(x_{j}\right), 1 \leq j \leq k$, and let $i_{j}$ be the $i \in\{1,2,3\}$ with $\sigma_{j} \in A_{i}$. We have $\operatorname{sgn}_{C}\left(x_{j}\right)=\operatorname{sgn}\left(\sigma_{j-1}, \sigma_{j}\right) \equiv i_{j-1}-i_{j} \bmod 3$, and so $s_{j} \equiv$ $i_{0}-i_{j} \bmod 3$. Setting $j=k$ gives the result. 
The integer $\frac{1}{3} \sum \operatorname{sgn}_{C}\left(x_{j}\right)$ will be denoted by $\operatorname{sgn}(C)$, and called the sign characteristic of the curve $C$.

Note that we have defined in Section 1 the surface $S$ by describing the star of every $x \in X$. For a fixed $x$ there exist cycles $\sigma_{i} \in A_{i}$ and triangles $t_{i}, 1 \leq i \leq 3$, such that the neighbouring elements of the cyclic sequence

$$
\left(\sigma_{1}, t_{2}, \sigma_{3}, t_{1}, \sigma_{2}, t_{3}\right)
$$

share an edge starting or terminating at $x$. Each $t_{i}$ is between $\sigma_{i-1}$ and $\sigma_{i+1}$, the oriented edge shared by $t_{i}$ and $\sigma_{i-1}$ is an incoming edge with respect to $x$, and the edge shared by $t_{i}$ and $\sigma_{i+1}$ an outcoming edge (cf. Figure 1).

Lemma 2.2. Let $x \in X$ be moved by $\sigma_{j} \in A_{j}, 1 \leq j \leq 3$. Consider the components of the star of $x$ that are induced by $\left(\sigma_{i}^{-1}(x), x, \sigma_{i^{\prime}}(x)\right)$, where $i, i^{\prime} \in\{1,2,3\}$. These components can be denoted by $\Gamma_{0}$ and $\Gamma_{1}$ in such a way that the following holds.

(i) If $\operatorname{sgn}\left(\sigma_{i}, \sigma_{i^{\prime}}\right)=0$, then $i=i^{\prime}, \Gamma_{0}=\left\{\sigma_{i}\right\}$ and $\Gamma_{1}=\left\{t_{i+1}, \sigma_{i-1}, t_{i}, \sigma_{i+1}, t_{i-1}\right\}$;

(ii) if $\operatorname{sgn}\left(\sigma_{i}, \sigma_{i^{\prime}}\right)=1$, then $i^{\prime} \equiv i-1 \bmod 3, \Gamma_{0}=\left\{\sigma_{i-1}, t_{i}, \sigma_{i+1}, t_{i-1}, \sigma_{i}\right\}$ and $\Gamma_{1}=\left\{t_{i+1}\right\} ;$ and

(iii) if $\operatorname{sgn}\left(\sigma_{i}, \sigma_{i^{\prime}}\right)=-1$, then $i^{\prime} \equiv i+1 \bmod 3, \Gamma_{0}=\left\{\sigma_{i+1}, t_{i-1}, \sigma_{i}\right\}$ and $\Gamma_{1}=$ $\left\{t_{i+1}, \sigma_{i-1}, t_{i}\right\}$.

Proof. The cyclic sequence before the lemma can be written in the form

$$
\left(\sigma_{i}, t_{i+1}, \sigma_{i-1}, t_{i}, \sigma_{i+1}, t_{i-1}\right) .
$$

The incoming edge $\left(\sigma_{i}^{-1}(x), x\right)$ is shared by $\sigma_{i}$ and $t_{i+1}$, while the outcoming edge is shared by either (i) $t_{i-1}$ and $\sigma_{i}$, or (ii) $t_{i+1}$ and $\sigma_{i-1}$, or (iii) $t_{i}$ and $\sigma_{i+1}$.

Corollary 2.3. Let $S$ be the combinatorial surface derived from $\left(\tau_{1}, \tau_{2}, \tau_{3}\right)$. Every closed polygonal curve has a border sequence consisting only of triangular faces, and a border sequence consisting only of cyclic faces.

Proof. Elements of both $\Gamma_{0}$ and $\Gamma_{1}$ appear in Lemma 2.2 in the order that is compatible with the cyclic ordering of the faces in the star of $x$. The starting and terminal faces of $\Gamma_{0}$ are always cyclic faces, while in $\Gamma_{1}$ the corresponding faces are triangular.

For every closed polygonal curve $C$ we hence have a triangle border sequence and a cycle border sequence. The elements of the former one will be known also as border faces. Note that a cycle appears in the latter one if and only if it belongs to the profile of $C$ (such cycles will be sometimes called profiled).

Suppose that $C$ is a separating closed polygonal curve. The component containing the triangle border sequence will be called the domain of $C$. (The domain is, seen formally, a combinatorial complex consisting of faces, edges and points.)

For any multigon $C$ let us denote by $\operatorname{Prof}(C)$ and $\operatorname{Vert}(C)$ the set of its profiled cycles, and the set of its vertices, respectively. If $C$ is separating, then we shall denote by $\operatorname{Cycl}(C)$ the set of cycles that induce a cyclic face in the domain of $C$. The set of those points in the domain that are not vertices of $C$ will be denoted by $\operatorname{Pnt}(C)$. 
Call a separating multigon planar if

$$
|\operatorname{Cycl}(C)|+\operatorname{sgn}(C)=|\operatorname{Pnt}(C)|+1 .
$$

It will become clear later that a simple multigon is planar if and only if its domain is homeomorphic to a disc. (We cannot start from such a definition, since we have to work with non-simple multigons as well.)

To exemplify the above notions let us turn again to the multigon with profile $\left(\alpha_{2}, \gamma_{3}\right.$, $\left.\alpha_{3}, \gamma_{1}, \beta_{4}, \alpha_{1}, \gamma_{4}\right)$ from Figure 2. The elements of the profile determine the cyclic faces that form the cyclic border sequence. The (triangular) border faces are $(g, l, n),(l, f, e)$, $(k, e, d),(j, d, c),(i, c, b),(h, b, a)$ and $(g, a, f)$. The vertices $g$ and $l$ are obtuse, and the other vertices are acute. The signature is thus equal to $(5-2) / 3=1$, the domain contains cyclic faces $\alpha_{4}, \gamma_{6}, \beta_{5}, \gamma_{5}, \beta_{6}$ and $\beta_{1}$, and $f, e, d, c, b$ and $a$ are the non-vertex points of the domain. We see that the multigon is really planar.

Let $C$ be a multigon with profile $\left(\sigma_{1}, \ldots, \sigma_{r}\right)$ and vertices $\left(v_{1}, \ldots, v_{r}\right)$. Then there clearly exists a unique multigon $C^{\prime}$ with profile $\left(\sigma_{r}, \ldots, \sigma_{1}\right)$ and vertices $\left(v_{r}, \ldots, v_{1}\right)$. The multigon $C^{\prime}$ will be called opposite, and will be denoted by $C^{\text {op }}$.

It is easy to describe all points of the multigon $C^{\text {op }}$. Choose $j, 1 \leq j \leq r$, and put $\sigma=\sigma_{j}$. Let $k$ be the length of $\sigma$. There exists a unique $h, 1 \leq h<k$, such that $v_{j+1}=\sigma^{h}\left(v_{j}\right)$. The sequence $v_{j} \sigma\left(v_{j}\right) \ldots \sigma^{h-1}\left(v_{j}\right) v_{j+1}$ yields the path of $C$ from $v_{j}$ to $v_{j+1}$. The path of $C^{\text {op }}$ from $v_{j+1}$ to $v_{j}$ is equal to $v_{j+1} \sigma\left(v_{j+1}\right) \ldots \sigma^{k-h-1}\left(v_{j+1}\right) v_{j}$.

Consider the sequence $\sigma\left(v_{j}\right) \ldots \sigma^{h-1}\left(v_{j}\right)$. If $\operatorname{sgn}_{C}\left(v_{j}\right)=-1$, add $v_{j}$ to the sequence as its initial element. If $\operatorname{sgn}_{C}\left(v_{j+1}\right)=-1$, add $v_{j+1}$ to the sequence as its terminal element. The resulting sequence is called the $\operatorname{arc}$ of $\sigma$ in $C$.

When working with $C$ and $C^{\mathrm{op}}$ at the same time, we sometimes call the $\operatorname{arc}$ of $\sigma$ in $C$ the inner arc, and the arc of $\sigma$ in $C^{\text {op }}$ the outer arc.

The inner arc contains the obtuse vertices, and excludes the acute vertices. By the definition of $\operatorname{sgn}_{C}\left(x_{j}\right)$ we see that a vertex is obtuse in $C$ if and only if it is acute in $C^{\text {op }}$. The following lemma is thus clear.

Lemma 2.4. Let $C$ be a multigon in $S$, and let $\sigma$ be one of its profiled cycles with the inner arc $x_{1} \ldots x_{s}$ and the outer arc $y_{1}, \ldots, y_{t}$. Then $\operatorname{sgn}(C)=-\operatorname{sgn}\left(C^{\text {op }}\right)$, and $\left(x_{1}, \ldots, x_{s}, y_{1}, \ldots, y_{t}\right)$ is the cyclic face of $\sigma$. In particular, $s+t=k$, where $k$ is the length of $\sigma$.

Suppose now that the multigon $C$ is separating, and consider the component of $C$ that contains the cyclic border sequence. Denote it by $\Gamma$. Note that the cyclic face induced by $\sigma=\sigma_{j}$ is repeated $h$ times in the sequence. A triangular face that has an edge induced by $\sigma$ is either a border face of $C$, or a border face of $C^{\text {op }}$. Remove from the component $\Gamma$ cyclic faces induced by all $\sigma_{j}, 1 \leq j \leq k$. We obtain a combinatorial complex, in which the edges with only one adjacent face are exactly the edges of $C^{\text {op }}$. Hence the complex coincides with the domain of $C^{\text {op }}$, and that makes the next statement obvious.

Proposition 2.5. Let $C$ be a multigon in the combinatorial surface $S$ of $\left(\tau_{1}, \tau_{2}, \tau_{3}\right)$. Assume that $S$ is connected. The multigon $C$ is separating if and only if the opposite multigon $C^{\mathrm{op}}$ is separating. In such a case each face of $S$ is either in the domain of $C$, or in the domain of $C^{\mathrm{op}}$, or it is a cyclic face determined by a profiled cycle. 
Proposition 2.6. Let $C$ be a separating planar multigon in the combinatorial surface $S$ of $\left(\tau_{1}, \tau_{2}, \tau_{3}\right)$. Suppose that $S$ is connected. If both $C$ and $C^{\mathrm{op}}$ are planar, then $S$ is a combinatorial sphere.

Proof. Let $T=\left(T^{\circ}, T^{*}\right)$ be the latin bi-trade determined by $\left(\tau_{1}, \tau_{2}, \tau_{3}\right)$. We wish to prove that $\operatorname{order}(T)=\operatorname{size}(T)+2$ (cf. Proposition 1.5). We have

$$
\operatorname{order}(T)=|\operatorname{Cycl}(C)|+\left|\operatorname{Cycl}\left(C^{\text {op }}\right)\right|+|\operatorname{Prof}(C)|
$$

and

$$
\operatorname{size}(T)=|\operatorname{Pnt}(C)|+\left|\operatorname{Pnt}\left(C^{\text {op }}\right)\right|+|\operatorname{Vert}(C)|,
$$

by Proposition 2.5. The multigons $C$ and $C^{\mathrm{op}}$ are assumed to be planar, which means that

$$
|\operatorname{Pnt}(C)|+\left|\operatorname{Pnt}\left(C^{\text {op }}\right)\right|+2=|\operatorname{Cycl}(C)|+\left|\operatorname{Cycl}\left(C^{\text {op }}\right)\right|+\operatorname{sgn}(C)+\operatorname{sgn}\left(C^{\text {op }}\right),
$$

by the definition. However, $\operatorname{sgn}(C)+\operatorname{sgn}\left(C^{\text {op }}\right)=0$ by Lemma 2.4 .

\section{Production rules}

In this section we shall present two rules that generate, when applied repeatedly, the set $\operatorname{Cycl}(C)$, where $C$ is a separating multigon in $S$. When the same procedure is used upon a non-separating multigon, then one gets the set of all cycles in the connectivity component of $C$.

Lemma 3.1. Let $C$ be a separating multigon with profile $\left(\sigma_{1}, \ldots, \sigma_{r}\right)$, and let $\sigma=\sigma_{j}$, $1 \leq j \leq r$. Any $\rho \in \operatorname{Cycl}(C)$ that meets $\sigma$ moves a point on the arc of $\sigma$. On the other hand, if a cycle $\rho$ moves a point on the arc of $\sigma$, then either $\rho \in \operatorname{Cycl}(C)$, or $\rho=\sigma_{k}$ for some $k \in\{1, \ldots, r\}$.

Proof. Suppose that $\rho \in \operatorname{Cycl} C$ meets $\sigma$ in a point $x$. Then $x$ is in the domain of $C$, and from Figure 3 we see that $x$ cannot be an acute vertex of $C$. Therefore $x$ has to belong to the arc of $\sigma$. Suppose on the other hand that a cycle $\rho$ meets $\sigma$ in an arc point $x$. Then $\rho$ has a common edge with a border triangle of $C$ (cf. Figure 3 again), and hence it belongs to $\mathrm{Cycl} C$ if not profiled.

Lemma 3.2. Let $C$ be a separating multigon. Cycles $\sigma$ and $\rho$ never meet when $\sigma \in$ $\operatorname{Cycl}(C)$ and $\rho \in \operatorname{Cycl}\left(C^{\mathrm{op}}\right)$. If both $\sigma$ and $\rho$ belong to the profile of $C$, then the inner arc of $\sigma$ never meets the outer arc of $\rho$.

Proof. The first part of the statement is easy. Suppose now that the inner arc of $\sigma \in$ $\operatorname{Prof}(C)$ meets the outer arc of $\rho \in \operatorname{Prof}(C)$ in a point $x$. We have $\rho \neq \sigma$ by Lemma 2.4, and hence $x$ is determined uniquely. Suppose first that $x$ is a vertex, and let $\rho^{\prime}$ and $\rho^{\prime \prime}$ be the two consecutive cycles of the profile that meet in $x$. We shall again refer to Figure 3. If $x$ is acute, then $\sigma \notin\left\{\rho^{\prime}, \rho^{\prime \prime}\right\}$, and the border triangles induced by $\sigma$ at $x$ belong to the domain of $C$. This is a contradiction since they also belong to the domain of $C^{\text {op }}$. 
Similarly, if $x$ is obtuse, then $\rho \notin\left\{\rho^{\prime}, \rho^{\prime \prime}\right\}$ induces at $x$ border triangles from the domain of $C^{\text {op }}$ that are also in $C$.

Suppose now that $x$ is not a vertex. Since we can consider $C^{\text {op }}$ in place of $C$, we see that $\operatorname{sgn}(\rho, \sigma)=1$ can be assumed. Thus $\left(\sigma(x), x, \rho^{-1}(x)\right)$ is a triangular face that is a border face of both $C$ and $C^{\text {op }}$.

To capture formally the relations appearing in Lemma 3.1, we shall define sets $\operatorname{In}(C)$ and $\operatorname{Out}(C), C$ a multigon in $S$, as sets of pairs of cycles that intersect in a predescribed way. Let $\left(\sigma_{1}, \ldots, \sigma_{r}\right)$ be the profile of $C$. For each $i \in\{1, \ldots, r\}$ define $\operatorname{In}\left(C, \sigma_{i}\right)$ as the set of all cycles $\rho \notin\left\{\sigma_{i-1}, \sigma_{i}, \sigma_{i+1}\right\}$ that meet the inner arc of $\sigma_{i}$. Put also $\overline{\operatorname{In}}\left(C, \sigma_{i}\right)=$ $\operatorname{In}\left(C, \sigma_{i}\right) \cup\left\{\sigma_{i-1}, \sigma_{i+1}\right\}$ and set $\operatorname{In}(C, \sigma)=\overline{\operatorname{In}}(C, \sigma)=\emptyset$ if $\sigma$ is not a profiled cycle. Define $\operatorname{In}(C)$ and $\overline{\operatorname{In}}(C)$ as the unions of all $(\rho, \sigma)$ such that $\rho \in \operatorname{In}(C, \sigma)$ and $\rho \in$ $\overline{\operatorname{In}}(C, \sigma)$, respectively. Instead of writing $\operatorname{In}\left(C^{\text {op }}, \sigma\right)$ we shall often be writing $\operatorname{Out}(C, \sigma)$, and this convention will be used for the other instances as well. Note that $\operatorname{In}\left(C, \sigma_{i}\right) \cup$ $\operatorname{Out}\left(C, \sigma_{i}\right) \cup\left\{\sigma_{i-1}, \sigma_{i+1}\right\}$ is a disjoint decomposition of all cycles that meet the cycle $\sigma_{i}$. This fact is illustrated by Figure 4 and will be used often further on.

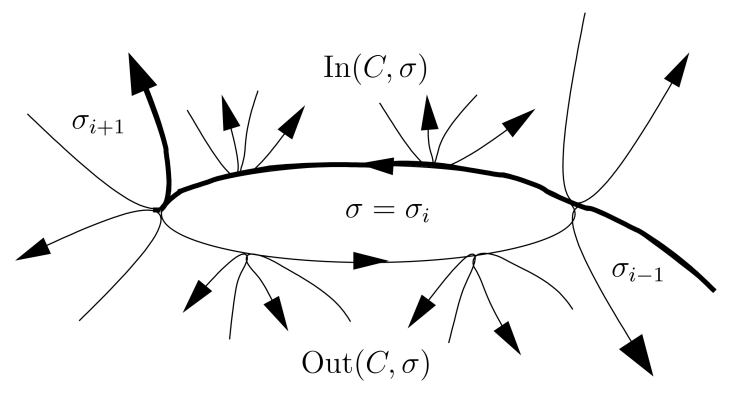

Figure 4. Cycles $\sigma_{i-1}$ and $\sigma_{i}$ meet in an obtuse vertex, while $\sigma_{i+1}$ and $\sigma_{i}$ in an acute vertex. The cycles that meet $\sigma_{i}$ above the bold line form $\operatorname{In}\left(C, \sigma_{i}\right)$.

For a set of cycles $\mathcal{N}$ consider the following rules:

(N1) If $(\rho, \sigma) \in \operatorname{In}(C)$ and $(\sigma, \rho) \notin \operatorname{In}(C)$, then $\rho \in \mathcal{N}$.

(N2) If $\rho \in \mathcal{N}$ meets a cycle $\sigma$ such that $(\rho, \sigma) \notin \operatorname{In}(C)$, then $\sigma \in \mathcal{N}$.

Here are several comments on the meaning of these rules. If $(\sigma, \rho) \notin \operatorname{In}(C)$, and $\sigma, \rho \in \operatorname{Prof}(C), \sigma \neq \rho$, then either $\rho=\sigma_{j \pm 1}$, or $(\sigma, \rho) \in \operatorname{Out}(C)$. The former option is excluded if we also assume $(\rho, \sigma) \in \operatorname{In}(C)$. Then $\rho$ and $\sigma$ meet both in the inner arc of $\sigma$, and in the outer arc of $\rho$. Inner and outer arcs do not meet when the multigon is separating by Lemma 3.2. Furthermore, if $C$ is separating, then $(\rho, \sigma) \in \operatorname{In}(C)$ implies $\rho \in \operatorname{Cycl}(C) \cup \operatorname{Prof}(C)$, by Lemma 3.1. We have proved

Lemma 3.3. Suppose that $(\rho, \sigma) \in \operatorname{In}(C)$ and $(\sigma, \rho) \notin \operatorname{In}(C)$. If $C$ is separating, then $\rho \in \operatorname{Cycl}(C)$. If $\rho \in \operatorname{Prof}(C)$, then the inner arc of $\sigma$ meets the outer arc of $\rho$, and $C$ is not separating. 
Let us now turn to rule (N2). Assume that $C$ is separating, $\rho \in \operatorname{Cycl}(C)$ meets $\sigma$, and $(\rho, \sigma) \notin \operatorname{In}(C)$. Lemma 3.1 states that if $\rho \in \operatorname{Cycl}(C)$ meets $\sigma \in \operatorname{Prof}(C)$, then $(\rho, \sigma) \in \operatorname{In}(C)$. Thus $\sigma \notin \operatorname{Prof}(C)$ and we easily get $\sigma \in \operatorname{Cycl}(C)$. Hence we can state

Lemma 3.4. If $C$ is a separating multigon, then the set $\mathcal{N}=\operatorname{Cycl}(C)$ is closed under the rules $(\mathrm{N} 1)$ and $(\mathrm{N} 2)$.

We also have

Lemma 3.5. Suppose that a set of cycles $\mathcal{N}$ is closed under the rules (N1) and (N2). If $\mathcal{N} \cap \operatorname{Prof}(C) \neq \emptyset$, then $\mathcal{N}$ contains all cycles in the connected component of $S$.

Proof. Consider $\rho \in \mathcal{N} \cap \operatorname{Prof}(C)$, and let $\sigma$ be the cycle immediately following $\rho$ in the profile of $C$. Then $(\rho, \sigma) \notin \operatorname{In}(C)$, and $\sigma \in \mathcal{N}$ by (N2). Hence $\operatorname{Prof}(C) \subseteq \mathcal{N}$, and it remains to show that a cycle $\sigma$ belongs to $\mathcal{N}$ whenever it meets some $\rho \in \mathcal{N}$. This follows from (N2) immediately since $\sigma \notin \operatorname{Prof}(C)$ can be assumed.

Let us have a nonempty collection of sets $\left(\mathcal{N}_{j} ; j \in J\right)$, and suppose that each set $\mathcal{N}_{j}$ is closed under the rules (N1) and (N2), for a given multigon $C$. Then $\bigcap_{j \in J} \mathcal{N}_{j}$ clearly preserves the rules as well, and so there exists the least set of cycles that is closed under (N1) and (N2).

Proposition 3.6. Let $C$ be a multigon in $S$, where the combinatorial surface $S$ is derived from $\left(\tau_{1}, \tau_{2}, \tau_{3}\right)$. Then there exists the least set of cycles $\mathcal{N}$ that is closed under the rules $(\mathrm{N} 1)$ and $(\mathrm{N} 2)$. If $C$ is separating, then this set $\mathcal{N}$ coincides with $\mathrm{Cycl}(C)$, and if it is not separating, then it is equal to the set of all cycles in the connected component of $S$.

Define $\mathcal{F}$ as a set of faces that consists of all

(F1) border faces of $C$;

(F2) cyclic faces induced by $\rho \in \mathcal{N}$; and

(F3) triangular faces that have an edge induced by $\rho \in \mathcal{N}$.

Then $\mathcal{F}$ is formed by all faces in the domain of $C$ when $C$ is a separating multigon. If it is not, then $\mathcal{F}$ coincides with the set of all faces in the connected component.

Proof. If $C$ is separating, then $\operatorname{Cycl}(C) \supseteq \mathcal{N}$ by Lemma 3.4, and thus all the faces of $\mathcal{F}$ are in the domain of $C$. We see that $\mathcal{F}$ is contained in exactly one block of the equivalence $\sim_{C}$ defined in Section 2. To prove the statement it suffices to show that $\mathcal{F}$ coincides with this block, by (F2). For that we need to verify the implication $F \in \mathcal{F} \Rightarrow G \in \mathcal{F}$ for all faces $F$ and $G$ that share an edge which is not an edge of $C$.

If $F$ is a cyclic face induced by $\rho$, then $\rho \in \mathcal{N}$ by (F2), and $G \in \mathcal{F}$, by (F3). Hence we can assume that $F$ is a triangular face. Note that to prove the case of $C$ non-separating it is enough to find any $\rho \in \operatorname{Prof}(C) \cap \mathcal{N}$, by Lemma 3.5.

Let first $F$ be a border face. Suppose that $\sigma$ induces a common edge of $F$ and $C$, and that $\rho$ induces the common edge of $F$ and $G$. The latter edge is not an edge of $C$, and hence $(\rho, \sigma) \in \operatorname{In}(C)$. Denote by $x$ the common point of $\sigma$ and $\rho$. Assume first $(\sigma, \rho) \in \operatorname{In}(C)$. Then $x$ is on the inner arc of $\rho$, and hence the other edge of $\rho$ that moves $x$ must be an edge of $C$. That implies $x \in \operatorname{Vert}(C)$. We have $(\sigma, \rho) \in \operatorname{In}(C)$, and so 
$\rho$ and $\sigma$ cannot be neighbours in the profile of $C$. Thus both $(x, \sigma(x))$ and $\left(\sigma^{-1}(x), x\right)$ are edges of $C$, and the common edge of $F$ and $G$ would be also an edge of $C$, if $x$ were an obtuse vertex (cf. Figure 2). Therefore $x$ is an acute vertex of $C$ and $(\sigma, \rho) \notin \operatorname{In}(C)$. Thus $\rho \in \mathcal{N}$ by (N1), and $G \in \mathcal{F}$ by (F2).

Assume now that the triangular face $F$ has an edge induced by some $\rho \in \mathcal{N}$, by (F3). Let $\sigma$ be the cycle that induces the common edge of $F$ and $G$, and let $x$ be the common point of $\sigma$ and $\rho$. Note that $G$ is in fact the cyclic face of $\sigma$. By the arguments above we can assume that $\rho \notin \operatorname{Prof}(C)$. If $(\rho, \sigma) \notin \operatorname{In}(C)$, then $\sigma \in \mathcal{N}$ by (N2), and so $G \in \mathcal{F}$, by (F2). To finish the proof it hence suffices to assume $(\rho, \sigma) \in \operatorname{In}(C)$ and to find a contradiction. That is not difficult since one sees easily from Figure 4 that under this assumption the common edge of $F$ and $G$ has to be also an edge of $C$.

In the following we shall often use the rules (N1) and (N2) in a way that implicitly refers to Proposition 3.6. The multigon $C$ will be understood from the context or will be explicitly declared. If $C$ will be separating, then by saying that a cycle belongs to $\operatorname{Cycl}(C)$ because of a rule $(\mathrm{N} 1)$ or $(\mathrm{N} 2)$ we shall mean that the rule can be applied to the case $\mathcal{N}=\operatorname{Cycl}(C)$.

\section{Triangle free multigons}

Let $C$ be a multigon with profile $\left(\sigma_{1}, \ldots, \sigma_{r}\right)$. Call $C$ triangle free if there exists no $j, 1 \leq j \leq r$, such that $\left(\sigma_{j-1}, \sigma_{j}, \sigma_{j+1}\right)$ or $\left(\sigma_{j+1}, \sigma_{j}, \sigma_{j-1}\right)$ is a profile of a triangle. Ultimately we are interested only in triangle free multigons. However to establish that there are enough of them in non-spherical trading surfaces we shall also need to work with multigons that are not triangle free. In this section we shall prove that each multigon either can be made triangle free by cutting and inserting stepwise the triangles that hinder the triangle freeness, or can be reduced by the same process to a single triangle. The construction is straightforward and is illustrated by Figure 5. For Lemma 4.1 use Figure 4. The formal proofs are omitted.

Lemma 4.1. Let $C$ be a multigon with profile $\left(\sigma_{1}, \ldots, \sigma_{r}\right)$. Put $\sigma=\sigma_{j}, 1 \leq j \leq r$, and define $\Sigma$ as the set of all cycles $\rho$ that meet $\sigma$. Order $\Sigma$ cyclically:

Let $\rho, \rho^{\prime} \in \Sigma$ meet $\sigma$ in $x$ and $x^{\prime}$, respectively. Then $\rho^{\prime}$ is an immediate successor to $\rho$ if and only if

(a) either $x=x^{\prime}$ and $\operatorname{sgn}(\sigma, \rho)>\operatorname{sgn}\left(\sigma, \rho^{\prime}\right)$;

(b) or $x^{\prime}=\sigma(x)$ and $\operatorname{sgn}(\sigma, \rho)<\operatorname{sgn}\left(\sigma, \rho^{\prime}\right)$.

Both $\operatorname{In}(C, \sigma)$ and $\overline{\operatorname{In}}(C, \sigma)$ are intervals in this cyclic ordering,

$$
\overline{\operatorname{In}}(C, \sigma)=\operatorname{In}(C, \sigma) \cup\left\{\sigma_{j-1}, \sigma_{j+1}\right\},
$$

and exactly one of the following cases holds:

(1) $\operatorname{In}(C, \sigma)=\emptyset$, and $\left(\sigma_{j-1}, \sigma_{j}, \sigma_{j+1}\right)$ is a profile of a triangle. The arc of $\sigma$ in $C$ is empty. 
(2) $\overline{\operatorname{In}}(C, \sigma)=\Sigma$ and $\left(\sigma_{j+1}, \sigma_{j}, \sigma_{j-1}\right)$ is a profile of a triangle. The arc of $\sigma$ in $C$ consists of all points moved by $\sigma$.

(3) Both $\operatorname{In}(C, \sigma)$ and $\overline{\operatorname{In}}(C, \sigma)$ are proper subsets of $\Sigma$, and none of the triples $\left(\sigma_{j-1}, \sigma_{j}\right.$, $\left.\sigma_{j+1}\right)$ and $\left(\sigma_{j+1}, \sigma_{j}, \sigma_{j-1}\right)$ is a triangle profile. Both the inner and outer arcs of $\sigma$ in $C$ are nonempty.

Corollary 4.2. Let $C$ be a multigon. Then $\operatorname{In}(C)=\emptyset$ if and only if $C$ is a triangle.

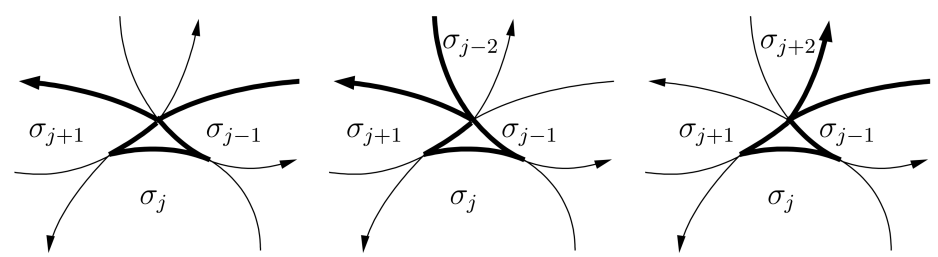

Figure 5. The three situations in which a border triangle is removed.

Lemma 4.3. Let $C$ be a multigon with profile $\left(\sigma_{1}, \ldots, \sigma_{r}\right)$ and vertices $\left(v_{1}, \ldots, v_{r}\right)$. Suppose that $C$ is not a triangle, and that $\operatorname{In}(C, \sigma)$ is empty for some $\sigma=\sigma_{j}, 1 \leq j \leq r$. Then $\sigma_{j-1}$ and $\sigma_{j+1}$ have a common point, say $x$, and $v_{j-1} \neq v_{j+2}$. There exists a unique multigon $C^{\prime}$ with a profile that is obtained from $\left(\sigma_{1}, \ldots, \sigma_{r}\right)$ by omitting

(1) $\sigma_{j}$ if $x \notin\left\{v_{j-1}, v_{j+2}\right\}$,

(2) $\sigma_{j-1}$ and $\sigma_{j}$, if $x=v_{j-1}$, and

(3) $\sigma_{j}$ and $\sigma_{j+1}$, if $x=v_{j+2}$.

If $C$ is separating, then $C^{\prime}$ is separating as well. In such a case the domain of $C^{\prime}$ is included in the domain of $C$, and $\left(x, v_{j+1}, v_{j}\right)$ is the only face in the domain of $C$ that does not belong to the domain of $C^{\prime}$.

Lemma 4.4 is intuitively clear. However, at this stage the notion of the planar multigon is not defined topologically, but through the equation $|\operatorname{Cycl}(C)|+\operatorname{sgn}(C)=|\operatorname{Pnt}(C)|+$ 1. By inspecting Figure 5 one can see that the respective equality remains true after the triangle is removed.

Lemma 4.4. Let $C$ and $C^{\prime}$ be multigons from Lemma 4.3. If $C$ (or $\left.C^{\mathrm{op}}\right)$ is planar, then $C^{\prime}\left(\right.$ or $\left.\left(C^{\prime}\right)^{\mathrm{op}}\right)$ is planar as well.

The procedure described in Lemma 4.3 can be repeated until there is no $j$ with $\operatorname{In}\left(C, \sigma_{j}\right)=\emptyset$. If $C$ is separating, then each of the modifications keeps $\operatorname{Cycl}(C)$ stable and increases $\operatorname{Cycl}\left(C^{\text {op }}\right)$ by a cycle from $\operatorname{Prof}(C)$. We can apply the same procedure to $C^{\text {op }}$, whenever $\operatorname{Out}\left(C, \sigma_{j}\right)=\emptyset$ for some $j$. Ultimately we obtain a multigon that is either triangle free, or is itself a triangle. We record this as

Proposition 4.5. Let $C$ be a polygon with profile $\left(\sigma_{1}, \ldots, \sigma_{r}\right)$. There exists a subsequence $1 \leq j_{r}<\cdots<j_{2} \leq r$ such that $\left(\sigma_{j_{1}}, \ldots, \sigma_{j_{r}}\right)$ is a profile of a multigon 
$C^{\prime}$ that is either a triangle, or a triangle free multigon. Furthermore, if $C$ is separating or planar, then $C^{\prime}$ has this property as well. If $C$ and $C^{\prime}$ are separating, then $\operatorname{Cycl}\left(C^{\prime}\right) \subseteq \operatorname{Cycl}(C) \cup \operatorname{Prof}(C)$ and $\operatorname{Cycl}\left(\left(C^{\prime}\right)^{\text {op }}\right) \subseteq \operatorname{Cycl}\left(C^{\text {op }}\right) \cup \operatorname{Prof}(C)$.

\section{Standard situations}

We shall often be dealing with two or three multigons that have common cycles which are positioned in a specific way. The purpose of this section is to describe several such positions for further reference. This will be done mostly in the language of $\operatorname{In}(C)$, Out $(C)$, $\overline{\operatorname{In}}(C)$ and $\overline{\operatorname{Out}}(C)$. The first two lemmas can be deduced directly from Figure 4.

Lemma 5.1. Let $C$ be a multigon with profile $\left(\sigma_{1}, \ldots, \sigma_{r}\right)$ and vertices $\left(v_{1}, \ldots, v_{r}\right)$. Assume $\sigma=\sigma_{j}, 1 \leq j \leq r$, and $\sigma^{h}\left(v_{j}\right)=v_{j+1}$, where $h$ is a positive integer less than the length of $\sigma$. Then $\left(\sigma^{i}\left(v_{j}\right), \sigma^{i+1}\left(v_{j}\right)\right), 1 \leq i<h$, are all edges of $C$ induced by $\sigma$. Each of the pairs $(\operatorname{In}(C, \sigma)$, Out $(C, \sigma))$ and $(\overline{\operatorname{In}}(C, \sigma), \overline{\operatorname{Out}}(C, \sigma))$ determines the integer $h$ and the points $\sigma_{j-1}^{-1}\left(v_{j}\right)$ and $\sigma_{j+1}\left(v_{j+1}\right)$. If $C$ is triangle free, then to determine these points and the integer $h$ it suffices to know only one of $\overline{\operatorname{In}}(C, \sigma), \operatorname{In}(C, \sigma), \operatorname{Out}(C, \sigma)$, and $\overline{\mathrm{Out}}(C, \sigma)$.

Lemma 5.2. Let $U$ and $V$ be multigons, and $\sigma \in \operatorname{Prof}(U) \cap \operatorname{Prof}(V)$. Consider the conditions

(a) $\operatorname{In}(U, \sigma) \supseteq \operatorname{In}(V, \sigma)$ and $\operatorname{Out}(V, \sigma) \supseteq \operatorname{Out}(U, \sigma)$, and

(b) $\operatorname{Out}(U, \sigma) \supseteq \operatorname{In}(V, \sigma)$ and $\operatorname{Out}(V, \sigma) \supseteq \operatorname{In}(U, \sigma)$.

The two inclusions in (a) as well as the inclusions in (b) are equivalent. The Conditions (a) and (b) are also equivalent to conditions obtained by replacing In and Out by $\overline{\mathrm{In}}$ and $\overline{\mathrm{Out}}$, respectively.

Say that a multigon $U$ wraps a multigon $V$ on $\sigma$ if $\sigma \notin \operatorname{Prof}(U) \cap \operatorname{Prof}(V)$ or Condition (a) holds. Say that $U$ wraps $V$ if $U$ wraps $V$ on every $\sigma \in \operatorname{Prof}(U)$ and $\operatorname{Prof}(U) \cap \operatorname{Prof}(V) \neq \emptyset$.

Say that a multigon $U$ abuts a multigon $V$ on $\sigma$ if $\sigma \notin \operatorname{Prof}(U) \cap \operatorname{Prof}(V)$ or Condition (b) holds. Say that $U$ abuts $V$ if $U$ abuts $V$ on every $\sigma \in \operatorname{Prof}(U)$ and $\operatorname{Prof}(U) \cap \operatorname{Prof}(V) \neq \emptyset$.

Lemma 5.3. Let $U$ and $V$ be multigons and $\sigma \in \operatorname{Prof}(U) \cap \operatorname{Prof}(V)$. The condition

$$
\overline{\operatorname{In}}(U, \sigma) \cap \operatorname{In}(V, \sigma)=\emptyset \quad \text { and } \quad \operatorname{In}(U, \sigma) \cap \overline{\operatorname{In}}(V, \sigma)=\emptyset
$$

is equivalent to the Condition (b) of Lemma 5.2. If both $U$ and $V$ are triangle free, then each of the equalities implies the other one.

Proof. Just note that $\operatorname{Out}(U, \sigma) \cup \overline{\operatorname{In}}(U, \sigma)$ is equal to the set $\Sigma$ of all $\rho \neq \sigma$ that meet $\sigma$. 
Corollary 5.4. Let $U$ and $V$ be multigons. Then $U$ abuts $V \Leftrightarrow V$ abuts $U \Leftrightarrow U^{\mathrm{op}}$ wraps $V \Leftrightarrow V^{\mathrm{op}}$ wraps $U$.

Say that multigons $U$ and $V$ complement each other on a cycle $\sigma$ if

(1) $U$ abuts $V$ on $\sigma$; and

(2) every edge induced by $\sigma$ is an edge of $U$ or $V$.

Note that an edge induced by $\sigma$ is never an edge of $U$ and $V$ simultaneously when $U$ abuts $V$ on $\sigma$. Note also that $\sigma \in \operatorname{Prof}(U) \cap \operatorname{Prof}(V)$ when $U$ complements $V$ on $\sigma$.

Say that a multigon $U$ divides into multigons $V$ and $W$ on $\sigma$ if

(1) $U$ wraps both $V$ and $W$ on $\sigma$;

(2) $V$ abuts $W$ on $\sigma$; and

(3) every edge of $U$ induced by $\sigma$ is an edge of $V$ or $W$.

Suppose that $U$ divides into $V$ and $W$ on $\sigma$. If $\sigma \notin \operatorname{Prof}(U)$, then the conditions just say that $\sigma \notin \operatorname{Prof}(V) \cup \operatorname{Prof}(W)$. Assume $\sigma \in \operatorname{Prof}(U)$. Then no edge of $U$ induced by $\sigma$ can be an edge of both $V$ and $W$. Note that if $\sigma \in \operatorname{Prof}(U)$, then $\sigma \in \operatorname{Prof}(V) \cup$ $\operatorname{Prof}(W)$, but not necessarily $\sigma \in \operatorname{Prof}(V) \cap \operatorname{Prof}(W)$. If $\sigma \in \operatorname{Prof}(U) \cap \operatorname{Prof}(V)$ and $\sigma \notin \operatorname{Prof}(W)$, then the edges of $U$ and $V$ that are induced by $\sigma$ overlap. Hence $U^{\text {op }}$ and $V$ have complementary edges, and we can use Lemma 5.4 to obtain

Lemma 5.5. Let $U, V$ and $W$ be multigons and suppose that $\sigma \in \operatorname{Prof}(U) \cap \operatorname{Prof}(V)$ is not a profiled cycle of $W$. Then $U$ divides into $V$ and $W$ on $\sigma$ if and only if $U^{\mathrm{op}}$ complements $V$ on $\sigma$.

If $U$ divides into $V$ and $W$ on $\sigma$, then clearly

$$
\operatorname{In}(U, \sigma) \subseteq \overline{\operatorname{In}}(V, \sigma) \cup \overline{\operatorname{In}}(W, \sigma) \subseteq \overline{\operatorname{In}}(U, \sigma) .
$$

Lemma 5.6. Let $\sigma^{\prime}$ and $\sigma^{\prime \prime}$ be neighbours of $\sigma$ in the profile of a multigon $U$. Suppose that $U$ divides into multigons $V$ and $W$ on $\sigma, \sigma^{\prime}$ and $\sigma^{\prime \prime}$. Suppose that $\rho \in \operatorname{In}(U, \sigma)$ does not belong to $\operatorname{In}(V, \sigma) \cup \operatorname{In}(W, \sigma)$. Let $x, v^{\prime}$ and $v^{\prime \prime}$ be the points where $\sigma$ meets $\rho, \sigma^{\prime}$ and $\sigma^{\prime \prime}$, respectively. Then $\rho$ neighbours $\sigma$ in the profile of $V$ or $W, x$ belongs to $\operatorname{Vert}(V) \cap \operatorname{Vert}(W)$, and

(1) either $x \in\left\{v^{\prime}, v^{\prime \prime}\right\}$ and $\operatorname{sgn}_{V}(x)=1=\operatorname{sgn}_{W}(x)$;

(2) or $x \notin\left\{v^{\prime}, v^{\prime \prime}\right\}$ and $\operatorname{sgn}_{V}(x)+\operatorname{sgn}_{W}(x)=0$;

(3) or $x \notin\left\{v^{\prime}, v^{\prime \prime}\right\}$ and $\operatorname{sgn}_{V}(x)=1=\operatorname{sgn}_{W}(x)$.

In Cases (1) and (2) the cycles $\rho$ and $\sigma$ are neighbours in profiles of both $V$ and $W$.

Proof. The inclusions before the lemma immediately imply $\rho \in \operatorname{Prof}(V) \cup \operatorname{Prof}(W)$, since $\rho \notin \operatorname{In}(V, \sigma) \cup \operatorname{In}(W, \sigma)$. If $\rho \in \overline{\operatorname{In}}(V, \sigma) \backslash \operatorname{In}(V, \sigma)$, then $\rho$ neighbours $\sigma$ in the profile of $V$, by the definition of $\operatorname{In}(V, \sigma)$. We see that $\rho$ neighbours $\sigma$ in one of the profiles.

Assume $x \notin\left\{v^{\prime}, v^{\prime \prime}\right\}$, and suppose that $\rho$ neighbours $\sigma$ in the profile of $V$. There are two edges of $U$ that are induced by $\sigma$ and contain $x$. Only one of them is an edge of $V$, as $x \in \operatorname{Vert}(V)$. The other one is hence an edge of $W$, and so one of Cases (2) and (3) clearly applies. 
Assume now $x \in\left\{v^{\prime}, v^{\prime \prime}\right\}$, say $x=v^{\prime}$. The edge of $U$ that contains $x$ and is induced by $\sigma$ is also an edge of $V$ or $W$. Let it be an edge of $V$. The other edge of $U$ that contains $x$ is induced by $\sigma^{\prime}$. It cannot be an edge of $V$, since then $x$ would appear twice as a vertex of $V$. Hence it is an edge of $W$, and $x$ is a vertex of $W$ since $U$ divides into $V$ and $W$ on $\sigma$. The cycle $\rho$ thus neighbours $\sigma^{\prime}$ in the profile of $W$, and we see that Case (1) applies.

Let us now turn to applications that involve separating multigons.

Proposition 5.7. Let $U$ and $V$ be separating multigons. If $U$ wraps $V$, then the domain of $U$ contains the domain of $V$.

Proof. All border faces of $V$ are clearly in the domain of $U$ if $\operatorname{Prof}(V) \subseteq \operatorname{Prof}(U) \cup$ $\operatorname{Cycl}(U)$. Hence it suffices to prove this inclusion and the inclusion $\operatorname{Cycl}(V) \subseteq \operatorname{Cycl}(U)$, by Proposition 3.6.

Let $\left(\mu_{1}, \ldots, \mu_{m}\right)$ be the profile of $U$, and $\left(\nu_{1}, \ldots, \nu_{n}\right)$ the profile of $V$. If $\nu_{j}=$ $\mu_{i} \in \operatorname{Prof}(U)$, then $\nu_{j+1}=\mu_{i+1}$ or $\nu_{j+1} \in \operatorname{In}\left(U, \mu_{i}\right)$, as $\overline{\operatorname{In}}\left(V, \mu_{i}\right) \subseteq \overline{\operatorname{In}}\left(U, \mu_{i}\right)$ by Lemma 5.2. In the latter case one can use for example Lemma 3.1 to obtain $\nu_{j+1} \in$ $\operatorname{Prof}(U) \cup \operatorname{Cycl}(U)$. If $\nu_{j} \in \operatorname{Cycl}(U)$, then clearly $\nu_{j+1} \in \operatorname{Prof}(U) \cup \operatorname{Cycl}(U)$, say by rule (N2). Hence $\operatorname{Prof}(V) \subseteq \operatorname{Prof}(U) \cup \operatorname{Cycl}(U)$ follows by induction on $j$. This also yields

$$
\operatorname{Prof}(U) \subseteq \operatorname{Prof}(V) \cup \operatorname{Cycl}\left(V^{\text {op }}\right)
$$

by Corollary 5.4. To finish we need to show that $\mathcal{N}=\operatorname{Cycl}(U) \cap \operatorname{Cycl}(V)$ is closed under rules (N1) and (N2) when $C=V$, since then $\operatorname{Cycl}(U) \cap \operatorname{Cycl}(V)=\operatorname{Cycl}(V)$ by Proposition 3.6.

Each of the rules yields an element of $\operatorname{Cycl}(V)$ by Proposition 3.6, and so our task rests in verifying that this element belongs to $\operatorname{Cycl}(U)$. In other words we wish to show that an instance of a rule (N1) or (N2) that is applicable to $\mathrm{Cycl}(V)$ can be used with respect to $\operatorname{Cycl}(U)$ as well. Since $\operatorname{Prof}(V) \subseteq \operatorname{Prof}(U) \cup \operatorname{Cycl}(U)$, this is clearly possible if we are able to avoid the non-presence clause. However, the non-presence clause never applies because the obtained cycle is in $\operatorname{Cycl}(V)$ and so it cannot be in $\operatorname{Prof}(U) \subseteq \operatorname{Prof}(V) \cup \operatorname{Cycl}\left(V^{\text {op }}\right)$.

Corollary 5.4 now yields a variant of the preceding proposition.

Proposition 5.8. Let $U$ and $V$ be separating multigons. If $U$ abuts $V$, then the domains of $U$ and $V$ have no common face.

\section{Decomposition of multigons}

Let $U, V$ and $W$ be multigons. Put $\Pi=(\operatorname{Prof}(V) \cup \operatorname{Prof}(W)) \backslash \operatorname{Prof}(U)$. Say that $U$ decomposes into $V$ and $W$ if

(1) $U$ divides into $V$ and $W$ on every $\sigma \in \operatorname{Prof}(U)$,

(2) $V$ complements $W$ on every $\rho \in \Pi$, and

(3) $\operatorname{Prof}(U) \cap \operatorname{Prof}(V) \neq \emptyset$ and $\operatorname{Prof}(U) \cap \operatorname{Prof}(W) \neq \emptyset$. 
Lemma 6.1. Suppose that $U$ decomposes into $V$ and $W$. Then $U$ wraps both $V$ and $W$, and $V$ abuts $W$. If $\left\{P_{1}, P_{2}, P_{3}\right\}=\{\operatorname{Prof}(U), \operatorname{Prof}(V), \operatorname{Prof}(W)\}$ and $\{i, j, k\}=$ $\{1,2,3\}$, then $P_{i} \cap P_{j} \neq \emptyset$ and $P_{j} \cup P_{k}=P_{i} \cup\left(P_{j} \cap P_{k}\right)$. In particular, $P_{i} \subseteq P_{j} \cup P_{k}$.

Proof. We shall first show $\operatorname{Prof}(V) \cap \operatorname{Prof}(W) \neq \emptyset$. Assume the contrary, and let $\left(\mu_{1}, \ldots, \mu_{n}\right)$ be the profile of $U$. We have $\mu_{1} \in \operatorname{Prof}(V) \cup \operatorname{Prof}(W)$; let us assume $\mu_{1} \in \operatorname{Prof}(V)$. If all $\mu_{i}$ belong to $\operatorname{Prof}(V)$, then $U=V$, which cannot be. We can hence find $\mu_{j} \in \operatorname{Prof}(V)$ with $\mu_{j+1} \notin \operatorname{Prof}(V)$. Then $\mu_{j+1} \in \operatorname{Prof}(W) \backslash \operatorname{Prof}(V)$, and $\mu_{j} \in \operatorname{Prof}(V) \backslash \operatorname{Prof}(W)$ by our assumptions. Let $\mu_{j}$ and $\mu_{j+1}$ intersect in the vertex $v$, and let $\rho$ be the third cycle that moves $v$. Then clearly $v \in \operatorname{Vert}(V) \cap \operatorname{Vert}(W)$, and $\rho \in \operatorname{Prof}(V) \cap \operatorname{Prof}(W)$.

The rest is easy. The multigon $U$ wraps both $V$ and $W$, by (1) and (3). We have $\Pi=(\operatorname{Prof}(V) \cap \operatorname{Prof}(W)) \backslash \operatorname{Prof}(U)$, by (2), and $\operatorname{Prof}(U) \subseteq \operatorname{Prof}(V) \cup \operatorname{Prof}(W)$, by (1). From that and from (3) one can deduce the assertions about $P_{1}, P_{2}$ and $P_{3}$. Finally, to see that $V$ abuts $W$ use (1), (2) and the fact that $\operatorname{Prof}(V) \cap \operatorname{Prof}(W) \neq \emptyset$.

Lemma 6.2. Suppose that $U$ decomposes into $V$ and $W$. Then $V^{\text {op }}$ decomposes into $U^{\text {op }}$ and $W$, and $W^{\mathrm{op}}$ decomposes into $U^{\mathrm{op}}$ and $V$.

Proof. We shall prove that $V^{\mathrm{op}}$ decomposes into $U^{\mathrm{op}}$ and $W$, and that means to verify Conditions (1-3) with respect to these multigons. From Lemma 6.1 we get immediately the validity of Condition (3). From the same lemma one also gets that $V^{\text {op }}$ wraps $U^{\text {op }}$ and that $V^{\text {op }}$ wraps $W$, by Corollary 5.4. By the same argument, $U^{\text {op }}$ abuts $W$. Note also that $\Pi^{\prime}=(\operatorname{Prof}(U) \cup \operatorname{Prof}(W)) \backslash \operatorname{Prof}(V)$ is equal to $(\operatorname{Prof}(U) \cap \operatorname{Prof}(W)) \backslash \operatorname{Prof}(V)$, by Lemma 6.1. If $\sigma \in \Pi^{\prime}$, then $U^{\text {op }}$ complements $W$ on $\sigma$, by Lemma 5.5, and so (2) holds.

For $\sigma \in \operatorname{Prof}(V)$ consider first the case $\sigma \notin \operatorname{Prof}(U)$. Then $V^{\text {op }}$ divides into $U$ and $W$ on $\sigma$, again by Lemma 5.5. Finally, let us have $\sigma \in \operatorname{Prof}(V) \cap \operatorname{Prof}(U)$. We need to show that an edge $e$ of $V^{\text {op }}$ that is induced by $\sigma$, is an edge of $U^{\text {op }}$ or an edge of $W$. If this were not true, then the edge $e$ would be an edge of $U$ and not an edge of $W$. But then it would be an edge of $V$, since $U$ divides into $V$ and $W$ on $\sigma$, a contradiction.

Lemma 6.3. Suppose that $U$ decomposes into $V$ and $W$. If $x$ is a vertex in one of the multigons, then it is a vertex in at least two of them. Set

$$
s(x)=\frac{1}{3}\left(\operatorname{sgn}_{V}(x)+\operatorname{sgn}_{W}(x)-\operatorname{sgn}_{U}(x)\right) .
$$

Then $s(x)=1$ if $x \in \operatorname{Vert}(U) \cap \operatorname{Vert}(V) \cap \operatorname{Vert}(W)$, and $s(x)=0$ in all other cases.

Proof. Note first that $3 s(x)=\operatorname{sgn}_{U^{\text {op }}}(x)+\operatorname{sgn}_{W}(x)-\operatorname{sgn}_{V^{\text {op }}}(x)$. Therefore one can assume $x \in \operatorname{Vert}(U)$, by Lemma 6.2. It is clear that $x \in \operatorname{Vert}(V) \cup \operatorname{Vert}(W)$, since $U$ divides into $V$ and $W$ on each $\sigma \in \operatorname{Prof}(U)$. Let $\sigma$ and $\sigma^{\prime}$ be the consecutive cycles in the profile of $U$ that meet at $x$. If $x$ appears as a vertex in only one of multigons $V$ and $W$, say in $V$, then $\sigma$ and $\sigma^{\prime}$ clearly have to be consecutive cycles in the profile of $V$ as well, and hence $\operatorname{sgn}_{V}(x)=\operatorname{sgn}_{U}(x)$. Suppose now that $x$ is a vertex of both $V$ and $W$, and let $\rho$ be the third cycle that moves $x$. Neither $V$ nor $W$ has $\sigma$ and $\sigma^{\prime}$ as consecutive cycles in their profiles since an edge of $V$ or $W$ that is induced by $\sigma$ or $\sigma^{\prime}$ has to be an 
edge of $U$. The pairs of consecutive cycles in the profiles hence have to be $(\sigma, \rho)$ and $(\rho, \sigma)$. Since $U$ wraps both $V$ and $W$, we must have $\operatorname{sgn}(\sigma, \rho)=1=\sigma\left(\rho, \sigma^{\prime}\right)$. Therefore $3 s(x)=1+1-(-1)=3$.

Proposition 6.4. Let $U, V$ and $W$ be multigons such that $U$ decomposes into $V$ and $W$. If any two of the multigons are separating, then the third multigon is separating as well.

Proof. By Lemma 6.2 and Proposition 2.5 it suffices to consider the case when $V$ and $W$ are separating multigons. We shall show that $\mathcal{N}=\Pi \cup \operatorname{Cycl}(V) \cup \operatorname{Cycl}(W)$ is closed under rules (N1) and (N2) when $C=U$, and that no element of $\operatorname{Prof}(U)$ belongs to $\mathcal{N}$. This will suffice, by Proposition 3.6.

Suppose that $(\rho, \sigma) \in \operatorname{In}(U)$ and $(\sigma, \rho) \notin \operatorname{In}(U)$. We wish to show $\rho \in \mathcal{N}$. Let us first consider the case $(\rho, \sigma) \in \operatorname{In}(V)$. If $(\sigma, \rho) \notin \operatorname{In}(V)$, then $\rho \in \operatorname{Cycl}(V)$ by rule (N1). Assume $(\sigma, \rho) \in \operatorname{In}(V)$. Then $\rho \in \operatorname{Prof}(V)$, and only the case $\rho \notin \Pi$ needs to be considered. In that case $\rho \in \operatorname{Prof}(U)$, and $(\sigma, \rho) \in \operatorname{In}(U)$ since $\operatorname{In}(V, \rho) \subseteq \operatorname{In}(U, \rho)$. However, this contradicts our assumptions. The case $(\rho, \sigma) \in \operatorname{In}(W)$ in similar, and hence we can assume $(\rho, \sigma) \notin \operatorname{In}(V) \cup \operatorname{In}(W)$. Then Lemma 5.6 applies, and we get $\rho \in \Pi$ in Cases (1) and (2). (This is immediate in Case (1), and follows from Lemma 6.3 in Case (2).) Let us consider Case (3). Denote by $x$ the intersecting point of $\sigma$ and $\rho$. Since $\operatorname{sgn}_{V}(x)=\operatorname{sgn}_{W}(x)=1$, by Lemma 5.6, there must be $\operatorname{sgn}_{U}(x)=-1$, by Lemma 6.3. The point $x$ is hence an intersecting point of consecutive cycles from the profile of $U$, say of $\sigma^{\prime}$ and $\sigma^{\prime \prime}$. The edges of $\sigma$ that move $x$ are edges of $C$, and hence $\sigma \notin\left\{\sigma^{\prime}, \sigma^{\prime \prime}\right\}$. The cycle $\rho$ thus equals $\sigma^{\prime}$ or $\sigma^{\prime \prime}$, and $x$ is on the inner arc of $\rho$ in $C$. Hence $(\sigma, \rho) \in \operatorname{In}(U)$, contrary to our assumption. We have verified that $\mathcal{N}$ is preserved by rule (N1).

When dealing with rule (N2) we shall consider apart cases $\rho \in \operatorname{Cycl}(V)$ and $\rho \in \Pi$. We assume that $\rho$ meets a cycle $\sigma$ such that $(\rho, \sigma) \notin \operatorname{In}(U)$.

If $\rho \in \operatorname{Cycl}(V)$ and $(\rho, \sigma) \notin \operatorname{In}(V)$, then $\sigma \in \operatorname{Cycl}(V)$, by (N2). If $(\rho, \sigma) \in \operatorname{In}(V)$, then $\sigma \notin \operatorname{Prof}(U)$, since $\operatorname{In}(V, \sigma) \subseteq \operatorname{In}(U, \sigma)$ for every $\sigma \in \operatorname{Prof}(U)$ and since we assume $(\rho, \sigma) \notin \operatorname{In}(U)$. But that means $\sigma \in \Pi \subseteq \mathcal{N}$.

Assume $\rho \in \Pi$, and denote by $x$ the intersection of $\rho$ and $\sigma$. Start by considering the case when $x$ is a point of the inner arc of $\rho$ in $V$. This means $(\sigma, \rho) \in \operatorname{In}(V)$. Suppose that $(\rho, \sigma) \in \operatorname{In}(V)$ as well. If $\sigma \in \Pi$, then $\sigma \in \mathcal{N}$, and so $\sigma \in \operatorname{Prof}(U)$ can be assumed. But that contradicts $\operatorname{In}(V, \sigma) \subseteq \operatorname{In}(U, \sigma)$ because $(\rho, \sigma) \notin \operatorname{In}(U)$. The case $(\rho, \sigma) \in \operatorname{In}(V)$ is thus clear, and the case $(\rho, \sigma) \notin \operatorname{In}(V)$ gives $\sigma \in \operatorname{Cycl}(V)$ by rule (N1). Therefore we can turn to the case when $x$ is a point on the inner arc of $\rho$ neither in $V$ nor in $W$. That means that $x$ is an acute vertex of both $V$ and $W$, since $V$ and $W$ complement each other on $\rho$. But then $x$ has to be an obtuse vertex $U$, by Lemma 6.3, and that implies $(\rho, \sigma) \in \operatorname{In}(U)$, a contradiction. We have verified that $\mathcal{N}$ is preserved by rule (N2).

Finally we have to refute the possibility that $\mathcal{N}$ consists of all cycles in the connectivity component. Note that only when this is done, one can conclude that Proposition 3.6 really guarantees that the multigon $U$ is separating.

The natural way seems to be to show that no $\sigma \in \operatorname{Prof}(U)$ belongs to $\mathcal{N}$. Assume the contrary. We cannot have $\sigma \in \Pi$ by the definition of $\Pi$, and so we can assume $\sigma \in \operatorname{Cycl}(V)$. This means $\sigma \in \operatorname{Prof}(W) \backslash \operatorname{Prof}(V)$ since $\operatorname{Prof}(V) \cap \operatorname{Cycl}(V)=\emptyset$. 
Consider now a triangular face with an edge of $W$ induced by $\sigma$. This is a border face of $W$ that is also a face in the domain of $V$, a contradiction to Proposition 5.8.

Proposition 6.5. Suppose that the multigon $U$ decomposes to multigons $V$ and $W$, and that all these multigons are separating. Then

$$
\operatorname{Cycl}(U)=\operatorname{Cycl}(V) \cup \operatorname{Cycl}(W) \cup \Pi \text {, where } \Pi=(\operatorname{Prof}(V) \cap \operatorname{Prof}(W)) \backslash \operatorname{Prof}(U) \text {. }
$$

The domain of $U$ contains the domains of both $V$ and $W$. Each face in the domain of $U$ is either in the domain of $V$, or in the domain of $W$, or it is a cyclic face induced by some $\sigma \in \Pi$.

Proof. We know from the proof of the previous proposition that $\operatorname{Cycl}(U)$ is contained in $\operatorname{Cycl}(V) \cup \operatorname{Cycl}(W) \cup \Pi$. On the other hand $\operatorname{Cycl}(U)$ contains both $\operatorname{Cycl}(V)$ and $\operatorname{Cycl}(W)$ by Proposition 5.7. We shall prove $\Pi \subseteq \operatorname{Cycl}(U)$.

Let $\left(\nu_{1}, \ldots, \nu_{m}\right)$ be the profile of $V$. We shall show that either $\nu_{j} \in \operatorname{Prof}(U)$, or $\nu_{j} \in \Pi \cap \operatorname{Cycl}(U)$. This can proceed by induction since $\operatorname{Prof}(U) \cap \operatorname{Prof}(V)$ is a nonempty set. If $\nu_{j+1} \in \operatorname{Prof}(U)$, then there is nothing to prove. Assume $\nu_{j+1} \in \Pi$, and let $x$ be the intersection point of $\nu_{j}$ and $\nu_{j+1}$. Let us first have $\nu_{j} \in \operatorname{Prof}(U)$. There cannot be $x \in \operatorname{Vert}(U) \backslash \operatorname{Vert}(W)$, as in such a case $\operatorname{sgn}_{V}(x)=\operatorname{sgn}_{U}(x)$ by Lemma 6.3, and that means $\nu_{j+1} \in \operatorname{Prof}(U)$. Assume $x \in \operatorname{Vert}(U) \cap \operatorname{Vert}(V)$. Then $x$ is an obtuse vertex of $U$, but an acute vertex of $V$, by Lemma 6.3. Hence $\left(\nu_{j+1}, \nu_{j}\right) \in \operatorname{In}(U)$, and $\left(\nu_{j}, \nu_{j+1}\right) \notin \operatorname{In}(U)$, since we can assume $\nu_{j+1} \notin \operatorname{Prof}(U)$. Therefore $\nu_{j+1} \in \operatorname{Cycl}(U)$, by rule (N1). We have solved the case $x \in \operatorname{Vert}(U)$. If $x \notin \operatorname{Vert}(U)$, then $x$ is a non-vertex point of $U$, and so $\left(\nu_{j+1}, \nu_{j}\right) \in \operatorname{In}(U)$, and rule (N1) can be applied again.

Suppose now that $\nu_{j} \in \Pi \cap \operatorname{Cycl}(U)$ and $\nu_{j+1} \in \Pi$. Then $\left(\nu_{j}, \nu_{j+1}\right) \notin \operatorname{In}(U)$, as $\nu_{j+1} \notin \operatorname{Prof}(U)$, and $\nu_{j+1} \in \operatorname{Cycl}(U)$ by rule (N2).

We have verified that $\operatorname{Cycl}(U)$ consists of $\operatorname{Cycl}(V), \operatorname{Cycl}(W)$ and $\Pi$. We know that the domain of $U$ contains the domains of $V$ and $W$, by Proposition 5.7. These domains have no common face by Proposition 5.8. The cyclic faces induced by elements of $\Pi$ belong to the domain of $U$, but not to the domain of $V$ or $W$, since $\Pi \subseteq \operatorname{Prof}(V) \cap$ $\operatorname{Prof}(W)$. Hence it remains to show that the faces in the domains of $V$ and $W$, plus the faces induced by elements of $\Pi$ really constitute all faces within the domain of $U$. For that we shall use Proposition 3.6. We already know that for the cyclic faces the assertion holds. Each triangular face adjacent to a cyclic face induced by $\sigma \in \Pi$ is a border face of $V$ or $W$, since $V$ complements $W$ on $\sigma$. Hence our set fulfils condition (F3). Each border face of $U$ is a border face of either $V$ or $W$, since $U$ divides into $V$ and $W$ on each $\sigma \in \operatorname{Prof}(U)$. The condition (F1) is hence fulfilled as well.

Proposition 6.6. Suppose that the multigon $U$ decomposes to multigons $V$ and $W$, and that two of the multigons $U, V$ and $W$ are planar. Put $\Pi=(\operatorname{Prof}(V) \cap \operatorname{Prof}(W)) \backslash$ $\operatorname{Prof}(U)$. If $|\Pi|+1=|\operatorname{Vert}(V) \cap \operatorname{Vert}(W)|$, then the third multigon is planar as well.

Proof. Put $\Delta_{C}=|\operatorname{Cycl}(V)|+|\operatorname{Cycl}(W)|-|\operatorname{Cycl}(U)|, \Delta_{s}=\operatorname{sgn}(V)+\operatorname{sgn}(W)-$ $\operatorname{sgn}(U)$ and $\Delta_{P}=|\operatorname{Pnt}(V)|+|\operatorname{Pnt}(W)|-|\operatorname{Pnt}(U)|$. The planarity of the third multigon is equivalent to

$$
\Delta_{C}+\Delta_{s}=\Delta_{P}+1 .
$$


Now, $\Delta_{C}=-|\Pi|$ by Proposition 6.5, and $\Delta_{s}=|\operatorname{Vert}(U) \cap \operatorname{Vert}(V) \cap \operatorname{Vert}(W)|$ by Lemma 6.3. Denote by $P(U), P(V)$ and $P(W)$ the sets $\operatorname{Pnt}(U) \cup \operatorname{Vert}(U), \operatorname{Pnt}(V) \cup$ $\operatorname{Vert}(V)$ and $\operatorname{Pnt}(W) \cup \operatorname{Vert}(W)$, respectively. We have $P(U)=P(V) \cup P(W)$, by Proposition 6.5. Furthermore, $P(V) \cap P(W)=\operatorname{Vert}(V) \cap \operatorname{Vert}(W)$, as $V$ and $W$ complement each other. Hence $|P(U)|+|\operatorname{Vert}(V) \cap \operatorname{Vert}(W)|=|P(V)|+|P(W)|$, and so $\Delta_{P}=|\operatorname{Vert}(V) \cap \operatorname{Vert}(W)|-|\operatorname{Vert}(V)|-|\operatorname{Vert}(W)|+|\operatorname{Vert}(U)|$. However,

$|\operatorname{Vert}(V)|=|\operatorname{Vert}(V) \cap \operatorname{Vert}(W)|+|\operatorname{Vert}(V) \cap \operatorname{Vert}(U)|-|\operatorname{Vert}(U) \cap \operatorname{Vert}(V) \cap(W)|$,

and for both $\operatorname{Vert}(U)$ and $\operatorname{Vert}(W)$ one can get corresponding expressions as well, by Lemma 6.3. Hence $\Delta_{P}=|\operatorname{Vert}(U) \cap \operatorname{Vert}(V) \cap \operatorname{Vert}(W)|-|\operatorname{Vert}(V) \cap \operatorname{Vert}(W)|$. The equality $\Delta_{C}+\Delta_{s}=\Delta_{P}+1$ thus holds if and only if $|\operatorname{Vert}(V) \cap \operatorname{Vert}(W)|=|\Pi|+1$.

\section{The existence of non-separating multigons}

Let $C$ be a multigon in the surface $S$ of $\left(\tau_{1}, \tau_{2}, \tau_{3}\right)$, and let $\left(\sigma_{1}, \ldots, \sigma_{r}\right)$ be the profile of $C$. Recall that the multigon is simple if and only if $\sigma_{i}$ meets $\sigma_{j}$ exactly when $j=i+1$, where $0 \leq i<j<r$. (We use here the convention that $\sigma_{0}=\sigma_{r}$.)

Simple multigons are simple oriented polygonal curves, or, as one would usually say, they are oriented polygons. However, an oriented polygon need not be a multigon, since a polygon can return to the same cyclic face twice. Note also that an oriented polygon that is a multigon need not be a simple multigon, since $\sigma_{i}$ and $\sigma_{j}$ can intersect in their outer arcs even when they do not intersect in a point of $C$.

In this section we shall show that the existence of a non-separating multigon implies the existence of a non-separating simple triangle free multigon.

Let $C$ be a multigon and $v$ a point of $S$. Denote by $\mathcal{E}_{v}(C)$ the set of all pairs $\left(e_{1}, e_{2}\right)$, where $e_{1}=\left(x_{1}, v\right)$ and $e_{2}=\left(v, x_{2}\right)$ are oriented edges of $S$ such that $x_{1}, v$ and $x_{2}$ are consecutive points of $C$. (This can be expressed also by saying that $e_{1}$ and $e_{2}$ are consecutive edges of $C$.)

Note that $0 \leq\left|\mathcal{E}_{v}(C)\right| \leq 3$, and that at most one pair $\left(e_{1}, e_{2}\right) \in \mathcal{E}_{v}(C)$ can have the property that the cycles inducing $e_{1}$ and $e_{2}$ are distinct.

Lemma 7.1. Let $U, C$ and $V$ be multigons, and let $v$ be a point of $S$ such that

$$
\mathcal{E}_{v}(C)=\mathcal{E}_{v}(U) \cup \mathcal{E}_{v}(V) \text { and } \quad \mathcal{E}_{v}(U) \cap \mathcal{E}_{v}(V)=\emptyset .
$$

Suppose that $U$ is separating and that $(e, f) \in \mathcal{E}_{v}(V)$. Denote by $E$ and $F$ the border faces of $V$ that are induced by $e$ and $f$, respectively.

If one of $E$ or $F$ belongs to the domain of $U$, then both of them are in the domain.

Proof. Let $\rho$ and $\rho^{\prime}$ be the cycles that induce $e$ and $f$, respectively. If $\operatorname{sgn}\left(\rho, \rho^{\prime}\right)=1$, then $E=F$, and nothing needs to be proved. If $E$ or $F$ is in the domain of $U$, then $\rho \in \operatorname{Cycl}(U)$ or $\rho^{\prime} \in \operatorname{Cycl}(U)$, respectively, since neither $e$ nor $f$ is an edge of $U$. The case $\rho=\rho^{\prime}$ is hence clear as well, and we can assume $\operatorname{sgn}\left(\rho, \rho^{\prime}\right)=-1$. Denote by $\sigma$ the third cycle that moves $v$, and note that $\sigma$ induces an edge of both $E$ and $F$. Denote these 
edges by $g$ and $h$, respectively. If $\sigma \in \operatorname{Cycl}(U)$, then both $E$ and $F$ are in the domain of $U$, and if $\sigma \in \operatorname{Cycl}\left(C^{\text {op }}\right)$, then none of them is in the domain. Assume $\sigma \in \operatorname{Prof}(U)$. We have $(e, f) \in \mathcal{E}_{v}(C)$, and hence either $(h, g) \in \mathcal{E}_{v}(C)$, or none of $g$ and $h$ is an edge of $C$. By our assumptions this means that either $(h, g) \in \mathcal{E}_{v}(U)$, or $(h, g) \in \mathcal{E}_{v}\left(U^{\text {op }}\right)$. Both $E$ and $F$ are in the domain of $U$ in the former case, and none of them is in the domain in the latter case.

In the next paragraphs we shall deal with the situation when $C$ is not a simple multigon, i.e. when $C$ contains a point that is repeated at least twice.

Let $x$ be the point. There must be two consecutive edges of $C$, both incident to $x$, that are induced by the same cycle, say $\sigma=\sigma_{j}$. (Otherwise $x$ would appear twice as a vertex, contrary to the definition of a multigon.) Since the point $x$ gets repeated, there must be another pair of consecutive edges of $C$ that are incident to $x$, say $\left(\rho^{-1}(x), x\right)$ and $\left(x, \rho^{\prime}(x)\right)$. We have $\rho=\sigma_{i}$ for some $i \neq j \pm 1$. The elements of the profile can be cyclically shifted, and hence we can assume $1 \leq i<j-1<r$, without loss of generality.

We shall define an oriented polygonal curve $V$ by its profile: the profile will be equal to $\left(\sigma_{i+1}, \ldots, \sigma_{j}\right)$ if $\rho^{\prime}=\sigma_{i+1}$, and to $\left(\sigma_{i}, \sigma_{i+1}, \ldots, \sigma_{j}\right)$ if $\rho^{\prime}=\sigma_{i}=\rho$. All edges of $V$ are also edges of $C$, and the edges $\left(\sigma^{-1}(x), x\right)$ and $\left(x, \rho^{\prime}(x)\right)$ are among them. The point $x$ is a vertex of $V$, and all other vertices of $V$ are vertices of $C$. The point $x$ is a vertex of $C$ if and only if $\rho \neq \rho^{\prime}$. We see that $V$ is a multigon.

Similar arguments can be used to show that there exists a multigon with profile $\left(\sigma_{1}\right.$, $\left.\ldots, \sigma_{i}, \sigma_{j}, \ldots, \sigma_{r}\right)$. This multigon will be denoted by $U$.

Suppose first that $\operatorname{sgn}\left(\rho, \rho^{\prime}\right)=1$ (i.e., that $x$ is an acute vertex of $C$ ) and that $U$ is separating. Let $v_{1} \ldots v_{k}$ be the point-wise description of $V$, with $x=v_{1}$ and $v_{k}=$ $\sigma^{-1}(x)$, and let $E$ be the triangular face with edges induced by $\rho$ and $\rho^{\prime}$. Since $\left(\rho^{-1}(x), x\right)$ is an edge of $U$ and $\left(x, \rho^{\prime}(x)\right)=\left(v_{1}, v_{2}\right)$ is an edge of $V$, we see that $E$ is a border face of both $U$ and $V$. In particular, $E$ is in the domain of $U$. Denote by $E_{i}, 1 \leq i \leq k$, the border face of $V$ determined by $\left(v_{i}, v_{i+1}\right)$.

If $v=v_{i+1}, 1 \leq i<k$, then the assumptions of Lemma 7.1 are satisfied, and we can prove, by induction, that all $E_{i}, 1 \leq i \leq k$, belong to the domain of $U$. The edge $\left(\sigma^{-1}(x), x\right)$ is an edge of both $E_{k}$ and $V$. Since it is an edge of $V$, it is not an edge of $U$. Since $E_{k}$ is the domain of $U$, there must be $\sigma \in \operatorname{Cycl}(U)$, a contradiction to $\sigma \in \operatorname{Prof}(U)$. We see that $\operatorname{sgn}\left(\rho, \rho^{\prime}\right) \leq 0$ whenever $U$ is separating.

Assume now $\operatorname{sgn}\left(\rho, \rho^{\prime}\right) \leq 0$. Put $\Pi=(\operatorname{Prof}(U) \cup \operatorname{Prof}(V)) \backslash \operatorname{Prof}(C)$. This set is clearly empty, and hence $|\Pi|+1=|\operatorname{Vert}(U) \cap \operatorname{Vert}(V)|$. We easily see that $C$ decomposes into $V$ and $W$. (The only condition which needs to be taken care of is the fact that $C$ divides into $U$ and $V$ on $\sigma$, and that follows from $\operatorname{sgn}\left(\rho, \rho^{\prime}\right) \leq 0$.)

Propositions 6.4 and 6.6 therefore imply

Lemma 7.2. If both multigons $U$ and $V$ are separating, then the multigon $C$ is separating as well. If both multigons $U$ and $V$ are planar, then the multigon $C$ is planar as well.

We shall now turn to the case when neither $C$ nor $C^{\text {op }}$ possesses a repeated point. In other words, we assume that both $C$ and $C^{\text {op }}$ are oriented polygons. Suppose however that $C$ is not a simple multigon. 
That means that some $\sigma=\sigma_{j} \in \operatorname{Prof}(C)$ meets with some $\rho=\sigma_{i} \in \operatorname{Prof}(C)$ in a point $y$ of $C$ in such a way that $\left(\sigma^{-1}(y), y\right)$ and $(y, \sigma(y))$ are not edges of $C$, while $\left(\rho^{-1}(y), y\right)$ and $\left(y, \rho^{\prime}(y)\right)$ are consecutive edges of $C$, where $\rho^{\prime}=\rho$ or $\rho^{\prime}=\sigma_{i+1}$. In particular, $i \neq j \pm 1$. Denote by $z$ and $x$ the points where $\sigma$ meets $\sigma_{j-1}$ and $\sigma_{j+1}$, respectively. Clearly, $x, z \in \operatorname{Vert}(C)$, and $y \notin\left\{x, z, \sigma_{j+1}(x), \sigma_{j-1}^{-1}(z)\right\}$. We can assume $1 \leq i<j-1<r$, with no loss of generality.

Let $V$ be the closed oriented polygonal curve with profile $\left(\sigma_{i+1}, \ldots, \sigma_{j}\right)$ when $\sigma_{i+1}=$ $\rho^{\prime} \neq \rho$, and profile $\left(\sigma_{i}, \ldots, \sigma_{j}\right)$ when $\sigma_{i}=\rho^{\prime}=\rho$. The edges of $V$ which are induced by $\sigma$ go from $z$ to $y$ over $x$, and hence form a single segment. All other edges of $V$ are edges of $C$. The point $y$ is a vertex of $V$, and other vertices of $V$ are vertices of $C$ (note however that $x$ is not a vertex of $V$ ). If $\rho=\rho^{\prime}$, then $y$ is not a vertex of $C$, and we see that $V$ is a multigon.

Similar arguments can be used to show that there exists a multigon with profile $\left(\sigma_{1}\right.$, $\left.\ldots, \sigma_{i}, \sigma_{j}, \ldots, \sigma_{r}\right)$. This multigon will be denoted by $U$.

Suppose now that the multigon $U$ is separating and that $\operatorname{sgn}(\rho, \sigma)=1$. Since $\sigma=\sigma_{j}$ moves points on the way from $y \in \operatorname{Vert}(U)$ to $x \in \operatorname{Vert}(U)$ through the point $z$, we see that triangular faces with edges $\left(\sigma^{-1}(z), z\right)$ and $(z, \sigma(z))$ are border faces of $U$. One of these faces has an edge moved by $\sigma_{j-1} \notin \operatorname{Prof}(U)$ and hence $\sigma_{j-1} \in \operatorname{Cycl}(U)$. Denote by $E$ the triangular face that contains the edge $\left(\sigma_{j-1}^{-1}(z), z\right)$. This is a border face of $V$ that is in the domain of $U$. By working against the arrows of $V$ we derive from Lemma 7.1 that the domain of $U$ contains also the border face of $V$ with the edge $\left(y, \rho^{\prime}(y)\right)$. That edge is not an edge of $U$. Denote the border face by $E^{\prime}$. If $\rho=\rho^{\prime}$, then we obtain $\rho^{\prime}=\sigma_{i} \in \operatorname{Cycl}(U)$, while $\rho^{\prime}=\sigma_{i+1}$ implies $\sigma_{j}=\sigma \in \operatorname{Cycl}(U)$, since $\left(\sigma^{-1}(y), y\right)$ is in this case another edge of $E^{\prime}$, and this edge is not an edge of $U$. We have obtained a contradiction in both cases since $\operatorname{Cycl}(U) \cap \operatorname{Prof}(U)=\emptyset$. Hence $\operatorname{sgn}(\rho, \sigma) \neq 1$ when $U$ is a separating multigon.

Assume now that $V$ is separating and $\operatorname{sgn}\left(\sigma, \rho^{\prime}\right)=1$. Starting from the border face $E$ of $U$ with edge $\left(x, \sigma_{j+1}(x)\right)$ we work along the arrows of $U$ up to the border face $E^{\prime}$ with the edge $\left(\rho^{-1}(y), y\right)$. The multigon $V$ yields edges $\left(\sigma^{-1}(x), x\right)$ and $(x, \sigma(x))$, and so $\sigma_{j+1} \notin \operatorname{Prof}(V)$. Hence $\sigma_{j+1} \in \operatorname{Cycl}(V)$, the triangle $E$ belongs to the domain of $V$, and $E^{\prime}$ belongs to the domain of $V$ by repeated applications of Lemma 7.1. By considering the triangular face $E^{\prime}$ we deduce that $\operatorname{Prof}(V) \cap \operatorname{Cycl}(V)$ contains $\sigma$ or $\rho$, which is a contradiction to the assumption that $V$ is separating. Thus $\operatorname{sgn}\left(\rho, \sigma^{\prime}\right) \leq 0$ when $V$ is a separating multigon.

Lemma 7.3. Suppose that both $U$ and $V$ are separating. Then $U$ decomposes into $C$ and $V^{\mathrm{op}}$, and $C$ is separating as well. If $U$ and $V^{\mathrm{op}}$ are planar, then $C$ is also planar.

Proof. First note that if $\rho=\rho^{\prime}$, then either $\operatorname{sgn}\left(\sigma, \rho^{\prime}\right)=1$ or $\operatorname{sgn}(\rho, \sigma)=1$. We have excluded both these alternatives, and hence there must be $\rho \neq \rho^{\prime}$ and $1=\operatorname{sgn}\left(\rho^{\prime}, \sigma\right)=$ $\operatorname{sgn}(\sigma, \rho)=\operatorname{sgn}\left(\rho, \rho^{\prime}\right)$. Polygons $U$ and $V^{\text {op }}$ have profiles $\left(\sigma_{1}, \ldots, \sigma_{i}, \sigma_{j}, \ldots, \sigma_{r}\right)$ and $\left(\sigma_{j}, \sigma_{j-1}, \ldots, \sigma_{i+1}\right)$, respectively. Thus $\Pi=(\operatorname{Prof}(V) \cup \operatorname{Prof}(C)) \backslash \operatorname{Prof}(U)=$ $\left\{\sigma_{i+1}, \ldots, \sigma_{j-1}\right\}$, where $\sigma_{i+1}=\rho^{\prime}, \sigma_{i}=\rho$ and $\sigma_{j}=\sigma$. It is now a straightforward task to verify that $U$ decomposes into $C$ and $V^{\text {op }}$, and that $\left|\operatorname{Vert}\left(V^{\text {op }}\right) \cap \operatorname{Vert}(C)\right|=|\Pi|+1$. The statement then follows from Propositions 6.4 and 6.6. 
Theorem 7.4. Let $S$ be a connected combinatorial surface determined by a triple of permutations $\left(\tau_{1}, \tau_{2}, \tau_{3}\right)$ of a set $X$ that satisfy conditions $(\mathrm{P} 1)$ and $(\mathrm{P} 2)$. Then $S$ is a combinatorial sphere if and only if every multigon of $S$ is separating. If $S$ is a combinatorial sphere, then every multigon of $S$ is planar. If $S$ is not a combinatorial sphere, then there exists a simple triangle free non-separating multigon.

Proof. Suppose first that there exists a non-separating multigon $C$ of profile $\left(\sigma_{1}, \ldots, \sigma_{r}\right)$. Choose $C$ in such a way that $r$ is minimal possible. If $C$ is not simple, consider $U$ and $V$ as defined in the earlier parts of this section. Both $U$ and $V$ have shorter profiles, and in Lemmas 7.2 and 7.3 we observed that $C$ has to be separating if both $U$ and $V$ are separating. The existence of $U$ and $V$ is thus excluded when $r$ is supposed to be the minimum among possible values of $|\operatorname{Prof}(C)|$.

Recall that Lemma 7.2 covered the case when $C$ is not a simple polygon. By transition to $C^{\mathrm{op}}$ it covers also the situation when $C^{\mathrm{op}}$ is not a simple polygon. The remaining possible violations of multigon simplicity of $C$ are treated by Lemma 7.3. Hence $C$ has to be a simple non-separating multigon. By Proposition 4.5, $C$ is triangle free.

In a combinatorial sphere there cannot exist a non-separating polygon, and so all multigons have to be separating if $S$ is a combinatorial sphere. If we find a multigon $C$ such that both $C$ and $C^{\text {op }}$ are planar, then $S$ is a combinatorial sphere by Proposition 2.6. To finish we thus need to prove that every multigon is planar whenever $S$ has the property that every multigon $C$ is separating.

Let $C$ be a counterexample with the least number of faces in the domain of $C$, and let $\left(\sigma_{1}, \ldots, \sigma_{r}\right)$ be the profile of $C$. From Lemma 7.2 we see that $C$ cannot have repeated points, and so it is a simple polygon. It cannot be a triangle since all triangles are planar, and hence $\operatorname{In}\left(C, \sigma_{j}\right) \neq \emptyset$ for every $j \in\{1, \ldots, r\}$, by Lemma 4.3. Therefore $\left(\sigma_{j-1}, \sigma_{j}, \sigma_{j+1}\right)$ is a profile of triangle for no $j \in\{1, \ldots, r\}$.

Suppose first $C$ contains an acute vertex, say $v_{j}$. Let $\rho$ be the cycle with $\sigma_{j-1} \rho \sigma_{j}\left(v_{j}\right)$ $=v_{j}$. The edge $\left(\sigma_{j}\left(v_{j}\right), \sigma_{j-1}^{-1}\left(v_{j}\right)\right)$ is not an edge of $C$, since $C$ is triangle free and without repeated points. Denote by $V$ the triangle $\sigma_{j-1}^{-1}\left(v_{j}\right) v_{j} \sigma_{j}\left(v_{j}\right)$, and by $W$ the polygon with profile

$$
\left(\sigma_{1}, \ldots, \sigma_{j^{\prime}}, \rho, \sigma_{j^{\prime \prime}}, \ldots, \sigma_{r}\right),
$$

where $j^{\prime} \in\{j-1, j-2\}$ and $j^{\prime \prime} \in\{j, j+1\}$ are chosen so that $j^{\prime}=j-2$ when $\sigma_{j-1}^{-1}\left(v_{j}\right)=v_{j-1}$ and $j^{\prime \prime}=j+1$ when $\sigma_{j}\left(v_{j}\right)=v_{j+1}$. We have obtained $W$ by "chopping" $V$ from $C$, and it is easy to see that $C$ decomposes into $V$ and $W$. We also see that the assumptions of Proposition 6.6 are fulfilled, and so $C$ is planar, a contradiction.

We can thus assume that all vertices of $C$ are obtuse. Instead of "chopping" a triangle of $C$, we shall "drag" it out of the domain. We shall describe the triangle $V$ and the multigon $W$, the rest then follows easily from Proposition 6.6, like in the previous case.

Choose $j, 1 \leq j \leq r$, and consider the cycles $\rho$ and $\rho^{\prime}$ with $\rho \rho^{\prime} \sigma_{j}\left(v_{j}\right)=v_{j}$. Let $V$ be the triangle with profile $\left(\sigma_{j}, \rho^{\prime}, \rho\right)$. We have $\rho^{\prime} \neq \sigma_{j+1}$ and $\rho \neq \sigma_{j-1} \operatorname{since} \operatorname{sgn}\left(\sigma_{j}, \rho^{\prime}\right)=$ $1=\operatorname{sgn}\left(\rho, \sigma_{j}\right)$. From the three edges of $V$ only the edge $\left(v_{j}, \sigma_{j}\left(v_{j}\right)\right)$ is an edge of $C$. Hence $\rho, \rho^{\prime} \in \operatorname{Cycl}(C)$, and one can define $W$ by the profile

$$
\left(\sigma_{1}, \ldots, \sigma_{j-1}, \rho, \rho^{\prime}, \sigma_{j^{\prime}}, \ldots, \sigma_{r}\right),
$$

where $j^{\prime} \in\{j, j+1\}$, with $j^{\prime}=j+1$ if and only if $\sigma_{j}\left(v_{j}\right)=v_{j+1}$. 


\section{Flip-flops}

Let $S$ be the combinatorial surface determined by $\left(\tau_{1}, \tau_{2}, \tau_{3}\right)$, where $\left(\tau_{1}, \tau_{2}, \tau_{3}\right)$ satisfies properties (P1) and (P2). Note first that the triple $\left(\tau_{2}^{-1}, \tau_{1}^{-1}, \tau_{3}^{-1}\right)$ satisfies the properties as well. Denote by $\overline{A_{i}}$ the set of cycles of $\tau_{i}^{-1}, 1 \leq i \leq 3$. Clearly $\rho \in \overline{A_{i}} \Leftrightarrow \rho^{-1} \in A_{i}$.

The arrows in $\bar{S}$, the combinatorial surface of $\left(\tau_{2}^{-1}, \tau_{1}^{-1}, \tau_{3}^{-1}\right)$, are exactly opposite to the arrows in $S$. Hence $x_{1} \ldots x_{k}$ is an oriented polygonal curve in $S$ whenever $x_{k} \ldots x_{1}$ is an oriented polygonal curve in $\bar{S}$. Thus for every multigon $C$ in $S$ of profile $\left(\sigma_{1}, \ldots, \sigma_{r}\right)$ we get a multigon $\bar{C}$ in $\bar{S}$ of profile $\left(\sigma_{k}^{-1}, \ldots, \sigma_{1}^{-1}\right)$. Since $\tau_{2}^{-1} \tau_{1}^{-1} \tau_{3}^{-1}$ is the identity mapping, we see that $\operatorname{sgn}\left(\rho^{-1}, \sigma^{-1}\right)=-\operatorname{sgn}(\rho, \sigma)$, for all cycles $\rho, \sigma \in$ $A_{1} \cup A_{2} \cup A_{3}, 1 \leq i \leq 3$. Therefore $\overline{\operatorname{sgn}}\left(\sigma_{j+1}^{-1}, \sigma_{j}^{-1}\right)=\operatorname{sgn}\left(\sigma_{j}, \sigma_{j+1}\right)$, and we see that $\overline{\operatorname{sgn}}_{\bar{C}}(v)=\operatorname{sgn}_{C}(v)$ for all $v \in \operatorname{Vert}(C)=\operatorname{Vert}(\bar{C})$. The inner arc of $\sigma_{j}$ in $C$ hence consists of exactly the same points as the inner arc of $\sigma_{j}^{-1}$ in $\bar{C}$.

There is nothing surprising in the facts above: when $\left(\tau_{1}, \tau_{2}, \tau_{3}\right)$ is seen as a latin bi-trade, then the transition to $\left(\tau_{2}^{-1}, \tau_{1}^{-1}, \tau_{3}^{-1}\right)$ corresponds to the exchange of rows and columns. It is sometimes worth to consider the additional symmetries offered by this transition, and we shall do so in this section.

We shall be describing a surgery on the surface $S$ that results in a combinatorial surface that can be interpreted as a latin bi-trade as well. There can be changes of the genus, and for that purposes we need to allow $S$ to have more components of connectivity (that is formally easier than considering a surgery that would interconnect different surfaces).

Let $C$ and $D$ be multigons in $S$. A mapping $f: \operatorname{Prof}(C) \rightarrow \operatorname{Prof}(D)$ will be called an isogony of $C$ onto $D$ if it is bijective, for every $\rho \in \operatorname{Prof}(C)$ the cycles $\rho$ and $f(\rho)$ belong to the same $A_{i}, 1 \leq i \leq 3$, the cycle $f\left(\rho^{\prime}\right)$ is consecutive to $f(\rho)$ in the profile of $D$ if $\rho^{\prime} \in \operatorname{Prof}(C)$ is consecutive to $\rho$, and in such a case $\operatorname{sgn}\left(\rho, \rho^{\prime}\right)=\operatorname{sgn}\left(f(\rho), f\left(\rho^{\prime}\right)\right)$. The multigons $C$ and $D$ are said to be isogonic if there exists at least one isogony $f$ : $\operatorname{Prof}(C) \rightarrow \operatorname{Prof}(D)$.

We shall assume that $C$ and $D$ have profiles $\left(\sigma_{1}, \ldots, \sigma_{r}\right)$ and $\left(\rho_{1}, \ldots, \rho_{r}\right)$, respectively, and that $f: \sigma_{j} \mapsto \rho_{j}$ is an isogony. The vertices will be denoted by $\left(v_{1}, \ldots, v_{r}\right)$ and $\left(w_{1}, \ldots, w_{r}\right)$. The isogony implies that $\operatorname{sgn}_{C}\left(v_{j}\right)=\operatorname{sgn}_{D}\left(w_{j}\right)$ for all $j, 1 \leq j \leq r$.

To define the surgery we shall have to impose several assumptions on $C$ and $D$. At the outset we shall assume that both $C$ and $D$ are triangle free and that $\sigma_{j} \neq \rho_{j}$ for every $j, 1 \leq j \leq r$.

Since $C$ and $D$ are triangle free, all inner and outer arcs are nonempty. For every $j$ define $a_{j}, b_{j}, c_{j}$ and $d_{j}$ in such a way that

$$
\sigma_{j}\left(c_{j}\right), \sigma_{j}^{2}\left(c_{j}\right), \ldots, a_{j} \quad \text { and } \quad \rho_{j}\left(d_{j}\right), \rho_{j}^{2}\left(d_{j}\right), \ldots, b_{j}
$$

are inner $\operatorname{arcs}$ of $C$ and $D$, respectively, and the outer arcs are

$$
\sigma_{j}\left(a_{j}\right), \sigma_{j}^{2}\left(a_{j}\right), \ldots, c_{j} \quad \text { and } \quad \rho_{j}\left(b_{j}\right), \rho_{j}^{2}\left(b_{j}\right), \ldots, d_{j} .
$$

Note that an inner arc or an outer arc can have only one point, so one has to consider cases $\sigma_{j}\left(c_{j}\right)=a_{j}$ etc. 
Suppose that $\sigma_{j}$ and $\rho_{j}$ belong to $A_{i}$. Then $\tau_{i}$ sends the terminal points of the arcs as follows:

$$
a_{j} \mapsto \sigma_{j}\left(a_{j}\right), \quad c_{j} \mapsto \sigma_{j}\left(c_{j}\right), \quad b_{j} \mapsto \rho_{j}\left(b_{j}\right) \quad \text { and } \quad d_{j} \mapsto \rho_{j}\left(d_{j}\right) .
$$

Define a new mapping $\tau_{i}^{\prime}$ in such a way that it differs from $\tau_{i}$ exactly on $a_{j}, c_{j}, b_{j}$ and $d_{j}$, for those $j, 1 \leq j \leq r$, such that $\sigma_{j}$ (and hence also $\rho_{j}$ ) belongs to $A_{i}$. The mapping $\tau_{i}^{\prime}$ maps the points $a_{j}, b_{j}, c_{j}$ and $d_{j}$ as follows:

$$
a_{j} \mapsto \rho_{j}\left(b_{j}\right), \quad c_{j} \mapsto \rho_{j}\left(d_{j}\right), \quad b_{j} \mapsto \sigma_{j}\left(a_{j}\right) \quad \text { and } \quad d_{j} \mapsto \sigma_{j}\left(c_{j}\right) .
$$

The mapping $\tau_{i}^{\prime}$ is clearly a permutation with cycles

$$
\left(\sigma_{j}\left(c_{j}\right) \sigma_{j}^{2}\left(c_{j}\right) \ldots a_{j} \rho_{j}\left(b_{j}\right) \rho_{j}^{2}\left(b_{j}\right) \ldots d_{j}\right),
$$

and

$$
\left(\rho_{j}\left(d_{j}\right) \rho_{j}^{2}\left(d_{j}\right) \ldots b_{j} \sigma_{j}\left(a_{j}\right) \sigma_{j}^{2}\left(a_{j}\right) \ldots c_{j}\right) .
$$

(The cycles of $\tau_{i}$ which do not appear in $\operatorname{Prof}(C) \cup \operatorname{Prof}(D)$ are also cycles of $\tau_{i}^{\prime}$, of course.) Let us label the above two cycles $\sigma_{j}^{\prime}$ and $\rho_{j}^{\prime}$, respectively.

We see that there exists a bijection $\hat{f}: \bigcup A_{i} \rightarrow \bigcup A_{i}^{\prime}$, where $A_{i}$ and $A_{i}^{\prime}$ are the cycle sets of $\tau_{i}$ and $\tau_{i}^{\prime}, 1 \leq i \leq 3$, that can be defined by $\hat{f}\left(\sigma_{j}\right)=\sigma_{j}^{\prime}, \hat{f}\left(\rho_{j}\right)=\rho_{j}^{\prime}, 1 \leq j \leq r$, and by $\hat{f}(\gamma)=\gamma$ when $\gamma \notin \operatorname{Prof}(C) \cup \operatorname{Prof}(D)$. Note that the definition of $\hat{f}$ does not depend, in fact, on the labelling of cycles that we use, but only on the isogony $f$.

The triple $\left(\tau_{1}^{\prime}, \tau_{2}^{\prime}, \tau_{3}^{\prime}\right)$ is said to be the flip-flop of $\left(\tau_{1}, \tau_{2}, \tau_{3}\right)$ by $f$. We shall also be saying that $\left(\tau_{1}^{\prime}, \tau_{2}^{\prime}, \tau_{3}^{\prime}\right)$ is the result of the flip-flop surgery along $f$.

Note that the mapping $\sigma_{j}^{-1} \mapsto \rho_{j}^{-1}$ yields an isogony $\bar{f}$ in $\bar{S}$, the surface determined by $\left(\tau_{2}^{-1}, \tau_{1}^{-1}, \tau_{3}^{-1}\right)$. The inner arc of $\sigma_{j}^{-1}$ in $\bar{S}$ is given by $a_{j}, \ldots, \sigma_{j}^{2}\left(c_{j}\right), \sigma_{j}\left(c_{j}\right)$, and the outer arc by $c_{j}, \ldots, \sigma_{j}^{2}\left(a_{j}\right), \sigma_{j}\left(a_{j}\right)$. We thus have $\overline{a_{j}}=\sigma_{j}\left(c_{j}\right)$ and $\overline{c_{j}}=\sigma_{j}\left(a_{j}\right)$. Similarly, $\overline{b_{j}}=\rho_{j}\left(d_{j}\right)$ and $\overline{d_{j}}=\rho_{j}\left(b_{j}\right)$. Assume $\sigma_{j}, \rho_{j} \in A_{i}, 1 \leq i \leq 3$. The equalities $\tau_{i}^{\prime}\left(a_{j}\right)=\rho_{j}\left(b_{j}\right), \tau_{i}^{\prime}\left(c_{j}\right)=\rho_{j}\left(d_{j}\right), \tau_{i}^{\prime}\left(b_{j}\right)=\sigma_{j}\left(a_{j}\right)$ and $\tau_{i}^{\prime}\left(d_{j}\right)=\sigma\left(c_{j}\right)$ can be thus expressed also in the form

$$
\begin{array}{llrl}
\left(\tau_{i}^{\prime}\right)^{-1}\left(\overline{d_{j}}\right)=a_{j}=\sigma_{j}^{-1}\left(\overline{c_{j}}\right), & & \left(\tau_{i}^{\prime}\right)^{-1}\left(\overline{b_{j}}\right)=c_{j}=\sigma_{j}^{-1}\left(\overline{a_{j}}\right), \\
\left(\tau_{i}^{\prime}\right)^{-1}\left(\overline{c_{j}}\right)=b_{j}=\rho_{j}^{-1}\left(\overline{d_{j}}\right) & \text { and } \quad & \left(\tau_{i}^{\prime}\right)^{-1}\left(\overline{a_{j}}\right)=d_{j}=\rho_{j}^{-1}\left(\overline{b_{j}}\right) .
\end{array}
$$

We see that the flip-flop surgery along $\bar{f}$ yields $\left(\left(\tau_{2}^{\prime}\right)^{-1},\left(\tau_{1}^{\prime}\right)^{-1},\left(\tau_{3}^{\prime}\right)^{-1}\right)$.

We have already observed that $\tau_{i}^{\prime}, 1 \leq i \leq 3$, are fixed point free permutations. Our goal now is to prove that $\tau_{1}^{\prime} \tau_{2}^{\prime} \tau_{3}^{\prime}$ is the identity mapping.

Suppose that $\tau_{1}^{\prime} \tau_{2}^{\prime} \tau_{3}^{\prime}(x) \neq x$ and $\tau_{3}^{\prime}(x)=\tau_{3}(x)$. Setting $x^{\prime}=\tau_{3}(x)$ yields $\tau_{3}^{\prime} \tau_{2}^{\prime} \tau_{1}^{\prime}\left(x^{\prime}\right)$ $\neq x^{\prime}$. This can be repeated until we find $i, 1 \leq i \leq 3$, and a point $y$ such that $\tau_{i+1}^{\prime} \tau_{i-1}^{\prime} \tau_{i}^{\prime}(y) \neq y$, and $\tau_{i}^{\prime}(y) \neq \tau_{i}(y)$. We thus need to prove $\tau_{i+1}^{\prime} \tau_{i-1}^{\prime} \tau_{i}^{\prime}(x)=x$ only for $x \in\left\{a_{j}, b_{j}, c_{j}, d_{j}\right\}, 1 \leq j \leq r$.

When the multigons $C$ and $D$ are exchanged, then the definition of $\tau_{i}^{\prime}, 1 \leq i \leq 3$, does not change. Transition to $C^{\mathrm{op}}$ and $D^{\mathrm{op}}$ does not influence it either. The inner and 
outer arcs are swapped in the latter transition, and so only the case $x=a_{j}$ needs to be considered. Let $\sigma_{j}$ be a cycle of $\tau_{i}, 1 \leq i \leq 3$.

Suppose first $\operatorname{sgn}\left(\sigma_{j}, \sigma_{j+1}\right)=1$. Then $v_{j+1}=\sigma_{j}\left(a_{j}\right)=c_{j+1}$ is an acute vertex of $C$. Hence $\tau_{i}^{\prime}\left(a_{j}\right)=\rho_{j}\left(b_{j}\right)=\sigma_{j}^{\prime}\left(a_{j}\right)=w_{j+1}$ is equal to $d_{j+1}$, and we obtain $\tau_{i-1}^{\prime} \tau_{i}^{\prime}\left(a_{j}\right)=$ $\tau_{i-1}^{\prime}\left(d_{j+1}\right)=\sigma_{j+1}^{\prime}\left(d_{j+1}\right)=\sigma_{j+1}\left(c_{j+1}\right)=\tau_{i-1}\left(c_{j+1}\right)=\tau_{i-1}\left(v_{j+1}\right)=\tau_{i-1} \tau_{i}\left(a_{j}\right)$. Therefore we need to show that $\tau_{i+1}$ agrees with $\tau_{i+1}^{\prime}$ on $\sigma_{j+1}\left(c_{j+1}\right)$.

Let $\gamma$ be the cycle of $\tau_{i+1}$ that moves the point $x=\sigma_{j+1}\left(c_{j+1}\right)$. If $\gamma \notin \operatorname{Prof}(C) \cup$ $\operatorname{Prof}(D)$, then $\tau_{i+1}$ and $\tau_{i+1}^{\prime}$ agree on $x$. We can hence assume that either (1) $\gamma=\sigma_{k}$ and $x \in\left\{a_{k}, c_{k}\right\}$, or (2) $\gamma=\rho_{k}$ and $x \in\left\{b_{k}, d_{k}\right\}$. In the former case $\{x, \gamma(x)\} \cap$ $\left\{v_{k}, v_{k+1}\right\} \neq \emptyset$, and in the latter case $\{x, \gamma(x)\} \cap\left\{w_{k}, w_{k+1}\right\} \neq \emptyset$.

Assume $\gamma(x)=v_{k+1}$ or $\gamma(x)=w_{k+1}$. Then $x=a_{k}$ or $x=b_{k}$, and $\gamma(x)$ is an acute vertex of $C$ or $D$, respectively. Since $\operatorname{sgn}\left(\gamma, \sigma_{j}\right)=1$, we get $\gamma=\sigma_{j-1}$ or $\sigma_{j}=\rho_{k+1}$. However $\gamma=\sigma_{j-1}$ contradicts the fact that $C$ is triangle free, and $\sigma_{j}=\rho_{k+1}$ contradicts $\operatorname{Prof}(C) \cap \operatorname{Prof}(D)=\emptyset$.

Assume $\gamma(x)=v_{k}$ or $\gamma(x)=w_{k}$. Then $x=c_{k}$ or $x=d_{k}$, and $\gamma(x)$ is an obtuse vertex of $C$ or $D$, respectively. Since $\operatorname{sgn}\left(\sigma_{j}, \gamma\right)=-1$, we get $\sigma_{j}=\sigma_{k-1}$ or $\sigma_{j}=\rho_{k-1}$. None of these equalities can hold.

Assume $x=v_{k+1}$ or $x=w_{k+1}$. Then $x=a_{k}$ or $x=b_{k}$, and $x$ is an obtuse vertex of $C$ or $D$, respectively. If $x=v_{k+1}$, then $\sigma_{j+1}=\sigma_{k+1}$, as $\operatorname{sgn}\left(\gamma, \sigma_{j+1}\right)=-1$, a contradiction to $\sigma_{j} \neq \gamma=\sigma_{k}$. If $x=w_{k+1}$, then $\sigma_{j+1}=\rho_{k+1}$, a contradiction to $\operatorname{Prof}(C) \cap \operatorname{Prof}(D)=\emptyset$.

Assume $x=v_{k}$ or $x=w_{k}$. Then $x=c_{k}$ or $x=d_{k}$, and $x$ is an acute vertex of $C$ or $D$, respectively. If $x=v_{k}$, then $\sigma_{k-1}=\sigma_{j+1}$ and $\gamma=\sigma_{j+2}$, which cannot be since $C$ is a triangle free multigon. If $x=w_{k}$, then $\gamma_{k-1}=\sigma_{j+1}$, a contradiction again.

It remains to consider the case $\operatorname{sgn}\left(\sigma_{j}, \sigma_{j+1}\right)=-1$. The cycles $\rho_{j+1}$ and $\sigma_{j+1}$ belong to $\tau_{i+1}, a_{j}=v_{j+1}=\sigma_{j+1}\left(c_{j+1}\right)$ and $b_{j}=w_{j+1}=\rho_{j+1}\left(d_{j+1}\right)$. Recall that $\rho_{j}\left(b_{j}\right)$ can be expressed as $\overline{d_{j}}$. We have $\rho_{j}^{-1}\left(\overline{d_{j}}\right)=b_{j}$ and $\overline{\operatorname{sgn}} \bar{D}\left(b_{j}\right)=-1$, as both $v_{j+1}$ and $w_{j+1}$ are obtuse vertices of $C$ and $D$, respectively. By turning from $C$ and $D$ to $\bar{C}$ and $\bar{D}$, one gets $\rho_{j}\left(b_{j}\right)$ in the role of $d_{j}$. Hence by turning from $C$ and $D$ to $\bar{D}^{\mathrm{op}}$ and $\bar{C}^{\mathrm{op}}$, one gets $\rho_{j}\left(b_{j}\right)$ in the role of $a_{j}$. Since $\rho_{j}^{-1}\left(\rho_{j}\left(b_{j}\right)\right)=b_{j}$ and $\overline{\operatorname{sgn}} \bar{D}^{\text {op }}\left(b_{j}\right)=1$ we can apply the preceding part of the proof to $\rho_{j}\left(b_{j}\right)$. We obtain

$$
\left(\tau_{i-1}^{-1}\right)^{\prime}\left(\tau_{i+1}^{-1}\right)^{\prime}\left(\tau_{i}^{-1}\right)^{\prime}\left(\rho_{j}\left(b_{j}\right)\right)=\rho_{j}\left(b_{j}\right),
$$

which is the same as

$$
\left(\tau_{i-1}^{\prime}\right)^{-1}\left(\tau_{i+1}^{\prime}\right)^{-1}\left(\tau_{i}^{\prime}\right)^{-1}\left(\rho_{j}\left(b_{j}\right)\right)=\rho_{j}\left(b_{j}\right),
$$

and so $\rho_{j}\left(b_{j}\right)=\tau_{i}^{\prime} \tau_{i+1}^{\prime} \tau_{i-1}^{\prime}\left(\rho_{j}\left(b_{j}\right)\right)$. Now, $\left(\tau_{i}^{\prime}\right)^{-1}\left(\rho_{j}\left(b_{j}\right)\right)=a_{j}$, and hence $a_{j}=$ $\tau_{i+1}^{\prime} \tau_{i-1}^{\prime} \tau_{i}^{\prime}\left(a_{j}\right)$, as required.

Theorem 8.1. Let $S$ be a combinatorial surface determined by permutations $\tau_{i}, 1 \leq i \leq$ 3 , such that $\left(\tau_{1}, \tau_{2}, \tau_{3}\right)$ fulfils conditions $(\mathrm{P} 1)$ and $(\mathrm{P} 2)$. Suppose that $C$ and $D$ are triangle free multigons in this surface.

Let $f$ be an isogony of $C$ onto $D$, and let $\left(\tau_{1}^{\prime}, \tau_{2}^{\prime}, \tau_{3}^{\prime}\right)$ be the result of the flip-flop surgery along $f$. Put $P=\operatorname{Prof}(C) \cup \operatorname{Prof}(D)$, and assume $\operatorname{Prof}(C) \cap \operatorname{Prof}(D)=\emptyset$. 
For $\gamma \in \operatorname{Prof}(C)$ put $g(\gamma)=f(\gamma)$, and denote by $I_{1}(\gamma)$ and $I_{-1}(\gamma)$ the inner and outer arcs of $\gamma$ in $C$. For $\gamma \in \operatorname{Prof}(D)$ put $g(\gamma)=f^{-1}(\gamma)$, and denote by $I_{1}(\gamma)$ and $I_{-1}(\gamma)$ the inner and outer arcs of $\gamma$ in $D$, respectively.

The triple $\left(\tau_{1}^{\prime}, \tau_{2}^{\prime}, \tau_{3}^{\prime}\right)$ fulfils conditions (P1) and (P2) if and only if the following holds:

(H1) Let $\gamma, \gamma^{\prime} \in P$ satisfy $\gamma^{\prime} \neq \gamma$ and $\gamma^{\prime} \neq g(\gamma)$. Then for any $\varepsilon, \eta \in\{-1,1\}$ at least one of the sets

$$
I_{\varepsilon}(\gamma) \cap I_{\eta}\left(\gamma^{\prime}\right) \quad \text { and } \quad I_{\varepsilon}(g(\gamma)) \cap I_{\eta}\left(g\left(\gamma^{\prime}\right)\right)
$$

is empty.

(H2) Let $\gamma, \gamma^{\prime} \in P$ satisfy $\gamma^{\prime} \neq \gamma$ and $\gamma^{\prime} \neq g(\gamma)$. Then for both $\varepsilon \in\{-1,1\}$ at least one of the sets

$$
I_{1}(\gamma) \cap I_{\varepsilon}\left(\gamma^{\prime}\right) \text { and } \quad I_{-1}(g(\gamma)) \cap I_{\varepsilon}\left(\gamma^{\prime}\right)
$$

is empty.

(H3) If $\gamma \in P$ and $\mu$ is a cycle, $\mu \notin P$, then $\mu$ never connects $I_{1}(\gamma)$ and $I_{-1}(g(\gamma))$.

Proof. Let $\mu$ be a cycle of $\tau_{i}^{\prime}$, and $\nu$ a cycle of $\tau_{i^{\prime}}^{\prime}$, where $i, i^{\prime} \in\{1,2,3\}$ and $i \neq i^{\prime}$. We have to show that $\mu$ and $\nu$ meet at most in one point.

If $\mu$ is a cycle of $\tau_{i}$ and $\nu$ of $\tau_{i^{\prime}}$, then they certainly meet at most in one point. We can hence assume that $\nu$ is not a cycle of $\tau_{i}$. By the construction of $\tau_{i}^{\prime}$ there exists $\gamma \in P$ such that the set of points moved by $\nu$ equals $I_{1}(\gamma) \cup I_{-1}(g(\gamma))$. By condition (H3), $\nu$ meets $\mu$ in at most one point when $\mu$ is cycle of $\tau_{i}$.

Let $I_{1}\left(\gamma^{\prime}\right) \cup I_{-1}\left(g\left(\gamma^{\prime}\right)\right)$ be the set of points moved by $\mu$, where $\gamma^{\prime} \in P$ and $\gamma^{\prime} \neq$ $\gamma$. Since both $\gamma$ and $\gamma^{\prime}$ move the points of $J_{1}=I_{1}(\gamma) \cap I_{1}\left(\gamma^{\prime}\right)$, this set contains at most one point. Similarly we see that each of the sets $J_{2}=I_{1}(\gamma) \cap I_{-1}\left(g\left(\gamma^{\prime}\right)\right), J_{3}=$ $I_{-1}(g(\gamma)) \cap I_{1}\left(\gamma^{\prime}\right)$ and $J_{4}=I_{-1}(g(\gamma)) \cap I_{-1}\left(g\left(\gamma^{\prime}\right)\right)$ has at most one element. The set $J=J_{1} \cup J_{2} \cup J_{3} \cup J_{4}$ coincides with the set of elements that are moved both by $\mu$ and $\nu$.

Condition (H2) expresses the fact that none of the pairs $\left(J_{1}, J_{2}\right),\left(J_{2}, J_{4}\right),\left(J_{3}, J_{4}\right)$ and $\left(J_{1}, J_{3}\right)$ consists of two nonempty sets. We also see that the condition (H2) is necessary. The set $J$ is hence equal to $J_{1} \cup J_{4}$ or to $J_{2} \cup J_{3}$. By condition (H1) none of the pairs $\left(J_{1}, J_{4}\right)$ and $\left(J_{2}, J_{3}\right)$ can consist of two nonempty sets, and hence $|J| \leq 1$. Note that condition (H1) does not change if one sets $\varepsilon=1$, and so we see easily that a violation of the condition yields a two element intersection.

We shall continue our discussion of the flip-flop surgery under the assumption that $f$ satisfies conditions (H1)-(H3). Hence $\left(\tau_{1}^{\prime}, \tau_{2}^{\prime}, \tau_{3}^{\prime}\right)$ yields a combinatorial surface and this surface will be denoted by $S^{\prime}$.

Let $\gamma$ and $\gamma^{\prime}$ be two distinct elements of $\operatorname{Prof}(C) \cup \operatorname{Prof}(D)$. The preceding proof shows that the sets $I_{1}(\gamma) \cup I_{-1}(g(\gamma))$ and $I_{1}\left(\gamma^{\prime}\right) \cup I_{-1}\left(g\left(\gamma^{\prime}\right)\right)$ have at most one common point. However, that also means that these sets are distinct, since each of $\tau_{i}^{\prime}, 1 \leq i \leq 3$, is a fixed point free permutations. Since these sets express the sets of points moved by cycles $\sigma_{j}^{\prime}$ and $\rho_{j}^{\prime}, 1 \leq j \leq r$, we see that $\sigma_{j}^{\prime} \neq \rho_{k}^{\prime}$ for all $j, k \in\{1, \ldots, r\}$, and that $\sigma_{j}^{\prime} \neq \sigma_{k}^{\prime}$ and $\rho_{j}^{\prime} \neq \rho_{k}^{\prime}$ if $1 \leq j<k \leq r$.

Fix now $j, 1 \leq j \leq r$. If $v_{j+1}$ is an obtuse vertex of $C$, then it is a common point of $\sigma_{j}^{\prime}$ and $\sigma_{j+1}^{\prime}$, while the common point of $\rho_{j}^{\prime}$ and $\rho_{j+1}^{\prime}$ is $w_{j+1}$. If $v_{j+1}$ is an acute vertex 
of $C$, then it is a common point of $\rho_{j}^{\prime}$ and $\rho_{j+1}^{\prime}$, while the common point of $\sigma_{j}^{\prime}$ and $\sigma_{j+1}^{\prime}$ is $w_{j+1}$.

From $\operatorname{Prof}(C) \cap \operatorname{Prof}(D)=\emptyset$ we get $\operatorname{Vert}(C) \cap \operatorname{Vert}(D)=\emptyset$, and so we see that each of the intersections of $\sigma_{j}^{\prime}$ and $\sigma_{j+1}^{\prime}$ is different from an intersection of $\sigma_{k}^{\prime}$ and $\sigma_{k+1}^{\prime}$, whenever $1 \leq j<k \leq r$. The same is true for $\rho_{j}^{\prime}$ and $\rho_{k}^{\prime}$, and so both $\left(\sigma_{1}^{\prime}, \ldots, \sigma_{r}^{\prime}\right)$ and $\left(\rho_{1}^{\prime}, \ldots, \rho_{r}^{\prime}\right)$ are profiles of multigons in $S^{\prime}$. Clearly $\operatorname{sgn}\left(\sigma_{j}, \sigma_{j+1}\right)=\operatorname{sgn}\left(\sigma_{j}^{\prime}, \sigma_{j+1}^{\prime}\right)=$ $\operatorname{sgn}\left(\rho_{j}, \rho_{j+1}\right)=\operatorname{sgn}\left(\rho_{j}^{\prime}, \rho_{j+1}^{\prime}\right)$.

The notion of isogony was defined for two multigons within the same surface but obviously it can be extended to a pair of multigons in two different surfaces. Recall that $\hat{f}$ has been defined to map naturally the cycles of $S$ upon the cycles of $S^{\prime}$.

Proposition 8.2. Let $S$ be a surface determined by $\left(\tau_{1}, \tau_{2}, \tau_{3}\right)$, and let $C$ and $D$ be triangle free multigons in $S$ such that $\operatorname{Prof}(C) \cap \operatorname{Prof}(D)=\emptyset$. Let $f$ be an isogony of $C$ onto $D$ that fulfils conditions (H1)-(H3). In the surface $S^{\prime}$ of $\left(\tau_{1}^{\prime}, \tau_{2}^{\prime}, \tau_{3}^{\prime}\right)$ there exist (uniquely determined) multigons $C^{\prime}$ and $D^{\prime}$ such that $\hat{f}$ yields isogonies of $C$ onto $C^{\prime}$, and $D$ onto $D^{\prime}$. These multigons are triangle free, $\operatorname{Prof}\left(C^{\prime}\right) \cap \operatorname{Prof}\left(D^{\prime}\right)=\emptyset$ and $\hat{f}(\sigma) \mapsto \hat{f}(f(\sigma))$, $\sigma \in \operatorname{Prof}(C)$, defines an isogony $f^{\prime}$ of $C^{\prime}$ onto $D^{\prime}$.

For $\sigma \in \operatorname{Prof}(C)$ put $\rho=f(\sigma)$ and denote by $I(\sigma), O(\sigma), I(\rho)$ and $O(\rho)$ the inner and outer arcs of $\sigma$ in $C$ and $\rho$ in $D$, respectively. Similarly, let $I^{\prime}(\sigma), O^{\prime}(\sigma), I^{\prime}(\rho)$ and $O^{\prime}(\rho)$ be the inner and outer arcs of $\hat{f}(\sigma)$ in $C^{\prime}$ and $\hat{f}(\rho)$ in $D^{\prime}$. Then $I^{\prime}(\sigma)=I(\sigma)$, $O^{\prime}(\sigma)=O(\rho), I^{\prime}(\rho)=I(\rho)$ and $O^{\prime}(\rho)=O(\sigma)$.

Proof. Nearly all statements of the proposition have been proved above. The part about arcs follows from the definition of $\tau_{i}^{\prime}, 1 \leq i \leq 3$, if we take into account that $C, C^{\prime}, D$ and $D^{\prime}$ are isogonic. The arcs in $C^{\prime}$ and $D^{\prime}$ are thus nonempty, and hence both $C^{\prime}$ and $D^{\prime}$ are triangle free.

Let $C^{\prime}$ and $D^{\prime}$, and the isogony $f^{\prime}$ of $C^{\prime}$ onto $D^{\prime}$ be the same as in Proposition 8.2.

Proposition 8.3. The isogony $f^{\prime}$ fulfils conditions $(\mathrm{H} 1)-(\mathrm{H} 3)$ with respect to $C^{\prime}$ and $D^{\prime}$. The flip-flop surgery of $\left(\tau_{1}^{\prime}, \tau_{2}^{\prime}, \tau_{3}^{\prime}\right)$ along $f^{\prime}$ yields $\left(\tau_{1}, \tau_{2}, \tau_{3}\right)$.

Proof. The relationships of arcs described in Proposition 8.2 imply that the flip-flop surgery along $f^{\prime}$ really yields $\left(\tau_{1}, \tau_{2}, \tau_{3}\right)$. The conditions (H1)-(H3) have to be fulfilled since in Theorem 8.1 we have proved that these conditions are necessary to obtain a triple that satisfies (P1)-(P2).

Theorem 8.4. Let $S$ be a combinatorial surface determined by permutations $\tau_{i}, 1 \leq i \leq$ 3 , such that $\left(\tau_{1}, \tau_{2}, \tau_{3}\right)$ fulfils conditions $(\mathrm{P} 1)$ and $(\mathrm{P} 2)$. Suppose that $C$ and $D$ are triangle free simple multigons in this surface.

Let $f$ be an isogony of $C$ onto $D$, and let $\left(\tau_{1}^{\prime}, \tau_{2}^{\prime}, \tau_{3}^{\prime}\right)$ be the result of the flip-flop surgery along $f$. Suppose that no cycle $\sigma \in \operatorname{Prof}(C)$ meets a cycle $\rho \in \operatorname{Prof}(D)$, and suppose that there is no cycle $\mu$ that connects the inner arc of $\sigma$ in $C$ with the outer arc of $\rho$ in $D$. Then $\left(\tau_{1}^{\prime}, \tau_{2}^{\prime}, \tau_{3}^{\prime}\right)$ fulfils $(\mathrm{P} 1)-(\mathrm{P} 2)$, the multigons $C^{\prime}$ and $D^{\prime}$ are simple, and no $\sigma^{\prime} \in \operatorname{Prof}\left(C^{\prime}\right)$ meets any $\rho^{\prime} \in \operatorname{Prof}\left(D^{\prime}\right)$. 
Proof. Note that condition (H2) is trivially true since cycles of $C$ and $D$ never meet. Condition (H1) is clearly satisfied when $\gamma \in \operatorname{Prof}(C)$ and $\gamma^{\prime} \in \operatorname{Prof}(D)$ by the same reason. We can hence assume $\gamma, \gamma^{\prime} \in \operatorname{Prof}(C)$. This can be further simplified to $\gamma=\sigma_{j}$ and $\gamma^{\prime}=\sigma_{j+1}$, as $C$ and $D$ are assumed to be simple. However, for this choice of $\gamma$ and $\gamma^{\prime}$ the condition (H2) always holds, since $f$ is an isogony. Condition (H3) is assumed explicitly in the text of theorem.

The triple $\left(\tau_{1}^{\prime}, \tau_{2}^{\prime}, \tau_{3}^{\prime}\right)$ thus satisfies (P1)-(P2), and we see that our assumptions imply that both $C^{\prime}$ and $D^{\prime}$ are simple, and that the cycles of $\operatorname{Prof}\left(C^{\prime}\right)$ do not meet the cycles of $\operatorname{Prof}\left(D^{\prime}\right)$.

\section{Flip-flops and separating multigons}

Throughout this section we shall assume that $\left(\tau_{1}, \tau_{2}, \tau_{3}\right)$ is a triple of permutations that satisfies (P1)-(P2), that $S$ is the combinatorial surface derived from $\left(\tau_{1}, \tau_{2}, \tau_{3}\right)$, that $C$ and $D$ are triangle free multigons in $S$ with $\operatorname{Prof}(C) \cap \operatorname{Prof}(D)=\emptyset$, and that $f$ is an isogony of $C$ onto $D$ that satisfies (H1)-(H3). Furthermore, by $\left(\tau_{1}^{\prime}, \tau_{2}^{\prime}, \tau_{3}^{\prime}\right)$ we shall denote the result of flip-flop surgery along $f$, by $S^{\prime}$ the combinatorial surface derived from $\left(\tau_{1}^{\prime}, \tau_{2}^{\prime}, \tau_{3}^{\prime}\right)$, and by $C^{\prime}$ and $D^{\prime}$ the multigons such that $\hat{f}$ induces an isogony of $C$ onto $C^{\prime}$, and of $D$ onto $D^{\prime}$.

When convenient we shall also assume that $\left(\sigma_{1}, \ldots, \sigma_{r}\right)$ and $\left(\rho_{1}, \ldots, \rho_{r}\right)$ are the profiles of $C$ and $D$, and that $\left(v_{1}, \ldots, v_{r}\right)$ and $\left(w_{1}, \ldots, w_{r}\right)$ are the vertices, respectively.

Note that neither $S$ nor $S^{\prime}$ needs to be a connected surface.

Proposition 9.1. Suppose that the multigon $C$ is separating and that $\operatorname{Prof}(D) \cap \operatorname{Cycl}(C)$ $=\emptyset$. Then $C^{\prime}$ is separating as well, $\operatorname{Cycl}(C)=\operatorname{Cycl}\left(C^{\prime}\right)$ and $\operatorname{Pnt}(C)=\operatorname{Pnt}\left(C^{\prime}\right)$. If $C$ is in addition planar, then $C^{\prime}$ is planar as well.

Proof. We shall start by showing that

$$
\operatorname{In}\left(C^{\prime}\right)=\{(\hat{f}(\rho), \hat{f}(\sigma)) ;(\rho, \sigma) \in \operatorname{In}(C)\} .
$$

Recall that $(\rho, \sigma) \in \operatorname{In}(C)$ if $\rho \neq \sigma$ meets $\sigma=\sigma_{j}$ in the inner arc of $\sigma, \rho \neq \sigma_{j \pm 1}$. If $(\rho, \sigma) \in \operatorname{In}(C)$, then $\rho \in \operatorname{Cycl}(C) \cup \operatorname{Prof}(C)$ by rule (N1). No $\rho \in \operatorname{Prof}(D)$ can thus meet $\sigma \in \operatorname{Prof}(C)$ in the inner arc of $\sigma$ since we assume $\operatorname{Prof}(D) \cap \operatorname{Cycl}(C)=\emptyset$. If $\left(\sigma_{j}, \sigma_{k}\right) \in \operatorname{In}(C)$, then $\sigma_{j}$ and $\sigma_{k}$ meet in the inner arcs by Lemma 3.2. We see that $\left(\sigma_{j}, \sigma_{k}\right) \in \operatorname{In}(C) \Leftrightarrow\left(\sigma_{j}^{\prime}, \sigma_{k}^{\prime}\right) \in \operatorname{In}\left(C^{\prime}\right)$ and that $\left(\rho_{j}^{\prime}, \sigma_{k}^{\prime}\right)$ is never in $\operatorname{In}\left(C^{\prime}\right)$, for all $j, k \in\{1,2, \ldots, r\}$. A cycle $\mu \notin \operatorname{Prof}(C) \cup \operatorname{Prof}(D)$ meets $\sigma_{j}$ in its inner arc if and only if it meets $\sigma_{j}^{\prime}$ in its inner arc, since the arcs coincide, and that finishes the verification of the formula for $\operatorname{In}\left(C^{\prime}\right)$.

Now put $\mathcal{N}=\hat{f}(\operatorname{Cycl}(C))$ and note that our expression of $\operatorname{In}\left(C^{\prime}\right)$ implies that $\mathcal{N}$ is closed by rule (N1), with respect to $C^{\prime}$. We shall prove that $\mathcal{N}$ is closed with respect to (N2) as well. If $\rho \in \operatorname{Cycl}(C)$ and $\sigma \in \bigcup A_{i}$ are such that $\hat{f}(\rho)$ meets $\hat{f}(\sigma)$, and $(\hat{f}(\rho), \hat{f}(\sigma)) \notin \operatorname{In}\left(C^{\prime}\right)$, then certainly $(\rho, \sigma) \notin \operatorname{In}(C)$. We do not have $\rho \in \operatorname{Prof}(C) \cup$ $\operatorname{Prof}(D)$, and so $\hat{f}(\rho)=\rho$. If $\rho$ meets $\sigma$, then $\sigma \in \operatorname{Cycl}(C)$ by rule (N2), and $\sigma=$ 
$\hat{f}(\sigma) \in \mathcal{N}$. It remains to show that $\rho$ really meets $\sigma$. This is clear when $\hat{f}(\sigma)=\sigma$. There cannot be $\sigma \in \operatorname{Prof}(D)$, since $\rho$ belongs to $\operatorname{Cycl}(C)$, and hence it neither meets some $\sigma_{k}$ in its outer arc, nor does it meet any $\rho_{k}$. If $\sigma=\sigma_{j}$, then $\rho$ meets $\sigma_{j}^{\prime}$. If the intersection is in the outer arc of $\sigma_{j}^{\prime}$, then $\rho$ meets $\rho_{j} \notin \operatorname{Prof}(C)$, and so $\rho_{j} \in \operatorname{Cycl}(C)$, a contradiction. Hence $\rho$ meets $\sigma=\sigma_{j}$ in every case. We have proved that $\mathcal{N}$ is closed under both (N1) and (N2). Thus $\hat{f}(\operatorname{Cycl}(C)) \supseteq \operatorname{Cycl}\left(C^{\prime}\right)$ by Proposition 3.6.

Since $C^{\prime}$ is separating we can use the reverse construction of Proposition 8.3 to show $\hat{f}^{-1}\left(\operatorname{Cycl}\left(C^{\prime}\right)\right) \supseteq \operatorname{Cycl}(C)$. Both inclusions together yield the equality $\operatorname{Cycl}\left(C^{\prime}\right)=$ $\hat{f}(\operatorname{Cycl}(C))$. Since $\operatorname{Cycl}(C)$ contains no element of $\operatorname{Prof}(C)$ or $\operatorname{Prof}(D)$, this means $\operatorname{Cycl}\left(C^{\prime}\right)=\operatorname{Cycl}(C)$.

Let $P$ and $P^{\prime}$ be sets of points that occur in domains of $C$ and $C^{\prime}$, respectively, and let $V$ and $V^{\prime}$ be the sets of acute vertices in $C$ and $C^{\prime}$, respectively. Then $P \backslash V$ consists of points moved by $\rho \in \operatorname{Cycl}(C)$ and of points on the inner arcs of $\sigma_{j}, 1 \leq j \leq r$. Hence $P \backslash V=P \backslash V^{\prime}$, which implies $\operatorname{Pnt}(C)=\operatorname{Pnt}\left(C^{\prime}\right)$. The multigons $C$ and $C^{\prime}$ are isogonic, and thus

$$
|\operatorname{Cycl}(C)|-|\operatorname{Pnt}(C)|+\operatorname{sgn}(C)=\left|\operatorname{Cycl}\left(C^{\prime}\right)\right|-\left|\operatorname{Pnt}\left(C^{\prime}\right)\right|+\operatorname{sgn}\left(C^{\prime}\right) .
$$

According to Proposition 8.2 the multigon $C^{\prime}$ is determined by isogony obtained from $\hat{f}$ by restricting the mapping to $\operatorname{Prof}(C)$.

Now put $f_{1}=f^{-1}$ and consider $f_{1}$ as an isogony of $C_{1}=D^{\text {op }}$ onto $D_{1}=C^{\text {op }}$. We know that the result of the flip-flop surgery along $f_{1}$ is $\left(\tau_{1}^{\prime}, \tau_{2}^{\prime}, \tau_{3}^{\prime}\right)$ again. Furthermore, $\widehat{f}_{1}\left(\rho_{j}\right)=\sigma_{j}^{\prime}$, as $\rho_{j}$ and $\sigma_{j}^{\prime}$ agree in the inner arcs of $C_{1}$ and $D_{1}$ (since they agree in the outer arcs of $C$ and $D$ ). By restricting $\widehat{f}_{1}$ to $C_{1}$ we see that $C_{1}$, which is of profile $\left(\rho_{r}, \ldots, \rho_{1}\right)$, determines $C_{1}^{\prime}$ as a polygon of profile $\left(\sigma_{r}^{\prime}, \ldots, \sigma_{1}^{\prime}\right)$. Thus $C_{1}^{\prime}=\left(C^{\prime}\right)^{\text {op }}$ and similarly $D_{1}^{\prime}=\left(D^{\prime}\right)^{\text {op }}$. Proposition 9.1 can thus alternatively be expressed in the following way:

Proposition 9.2. Suppose that the multigon $D$ is separating and that we have $\operatorname{Prof}(C) \cap$ $\operatorname{Cycl}\left(D^{\text {op }}\right)=\emptyset$. Then $C^{\prime}$ is separating as well, $\operatorname{Cycl}\left(\left(C^{\prime}\right)^{\text {op }}\right)=\operatorname{Cycl}\left(D^{\text {op }}\right)$ and $\operatorname{Pnt}\left(D^{\text {op }}\right)$ $=\operatorname{Pnt}\left(\left(C^{\prime}\right)^{\mathrm{op}}\right)$. If $D^{\mathrm{op}}$ is in addition planar, then $\left(C^{\prime}\right)^{\mathrm{op}}$ is planar as well.

We shall now bind together the propositions above.

Proposition 9.3. Suppose that both multigons $C$ and $D$ are separating and that

$$
\operatorname{Prof}(D) \cap \operatorname{Cycl}(C)=\emptyset=\operatorname{Prof}(C) \cap \operatorname{Cycl}\left(D^{\text {op }}\right) .
$$

Then $C^{\prime}$ is separating, $\operatorname{Cycl}\left(C^{\prime}\right)=\operatorname{Cycl}(C), \operatorname{Cycl}\left(\left(C^{\prime}\right)^{\text {op }}\right)=\operatorname{Cycl}\left(D^{\text {op }}\right)$, and the connected component of $C^{\prime}$ does not contain the cyclic faces induced by the profile of $D^{\prime}$. If both $C$ and $D^{\mathrm{op}}$ are planar multigons, then the connected component of $C^{\prime}$ is spherical.

Proof. The domains of $C^{\prime}$ and $\left(C^{\prime}\right)^{\text {op }}$ have their structure determined by Propositions 9.1 and 9.2. The cyclic faces of their connecting component are exactly those that are induced by an element of $\operatorname{Cycl}\left(C^{\prime}\right) \cup \operatorname{Cycl}\left(\left(C^{\prime}\right)^{\text {op }}\right) \cup \operatorname{Prof}\left(C^{\prime}\right)$, as follows from Proposition 2.5. None of them is an element of $\operatorname{Prof}\left(D^{\prime}\right)$, as $\operatorname{Cycl}\left(C^{\prime}\right)=\operatorname{Cycl}(C)$ and 
$\operatorname{Cycl}\left(\left(C^{\prime}\right)^{\text {op }}\right)=\operatorname{Cycl}\left(D^{\text {op }}\right)$. If $C$ and $D^{\text {op }}$ are planar, then $C^{\prime}$ and $\left(C^{\prime}\right)^{\text {op }}$ are planar as well, by Propositions 9.1 and 9.2, and the component is spherical by Proposition 2.6.

Proposition 9.4. Suppose that the multigon $C$ is separating, and that we have $\operatorname{Prof}(D) \cap$ $\operatorname{Cycl}\left(C^{\mathrm{op}}\right)=\emptyset$. If $D$ is not separating, then $C^{\prime}$ and $D^{\prime}$ are in the same connected component of $S^{\prime}$.

Proof. Since $D$ is not separating, there exists a way how to obtain an element of $\operatorname{Prof}(D)$ by means of rules (N1) and (N2) (see Proposition 3.6). By considering a sequence of the shortest length that produces such an element, we obtain a sequence $\gamma_{0}, \ldots, \gamma_{t}$ of cycles, $t \geq 1$, such that $\gamma_{0} \in \operatorname{Prof}(D), \gamma_{t} \in \operatorname{Prof}(D), \gamma_{i} \notin \operatorname{Prof}(D)$ if $1 \leq i<t$, and such that $\gamma_{1}$ is obtained from $\gamma_{0}$ by means of rule (N1), while $\gamma_{i}, t \geq i>1$, are obtained from $\gamma_{i-1}$ by means of rule $(\mathrm{N} 2)$.

Let us have $\gamma_{0}=\rho_{j}$ and $\gamma_{t}=\rho_{k}$. If $t=1$, then the inner arc of $\rho_{j}$ meets the outer arc of $\rho_{k}$ by Lemma 3.3, and so $\rho_{j}^{\prime}$ meets $\sigma_{k}^{\prime}$. Hence $t>1$ can be assumed.

Suppose first that none of elements $\gamma_{1}, \ldots, \gamma_{t-1}$ belongs to the profile of $C$. Then each of $\gamma_{1}, \ldots, \gamma_{t-1}$ is a cycle of $\tau_{1}^{\prime}$ or $\tau_{2}^{\prime}$ or $\tau_{3}^{\prime}$. Note that $\gamma_{1}$ meets $\gamma_{0}=\rho_{j}$ in the inner arc of $\rho_{j}$ by rule (N1), while $\gamma_{t-1}$ meets $\gamma_{t}=\rho_{k}$ in the outer arc of $\rho_{k}$, by rule (N2). Therefore $\rho_{j}^{\prime}$ meets $\gamma_{1}$ and $\gamma_{t-1}$ meets $\sigma_{k}^{\prime}$. We see that $C^{\prime}$ and $D^{\prime}$ are in the same component.

Let there exist $t^{\prime}, 1 \leq t^{\prime}<t$, with $\gamma_{t^{\prime}} \in \operatorname{Prof}(C)$, say $\gamma_{t^{\prime}}=\sigma_{s}$. Choose $t^{\prime}$ to be the least possible. If $\gamma_{t^{\prime}-1}$ meets $\sigma_{s}$ in the inner arc of $\sigma_{s}$, then we obtain a path from $\rho_{j}^{\prime}$ to $\sigma_{s}^{\prime}$. If $\rho_{t^{\prime}-1}$ meets $\sigma_{s}$ in the outer arc of $\sigma_{s}$, then, by working backwards along rules (N1) and (N2), we get $\gamma_{0}=\rho_{j} \in \operatorname{Cycl}\left(C^{\text {op }}\right)$, a contradiction to our assumptions.

We conclude this section by an easy lemma which does not seem to require a proof.

Lemma 9.5. Suppose that each connected component of $S$ contains $C$ or $D$. Then each connected component of $S^{\prime}$ contains $C^{\prime}$ or $D^{\prime}$.

\section{Triangular construction}

Let $\left(\tau_{1}, \tau_{2}, \tau_{3}\right)$ be a triple of permutations that satisfies $(\mathrm{P} 1)-(\mathrm{P} 2)$, and suppose that $\mathbf{x}=$ $x_{3} x_{2} x_{1}$ is a triangle in the combinatorial surface $S$ determined by $\left(\tau_{1}, \tau_{2}, \tau_{3}\right)$. We can assume that $\tau_{i}$ induces the edge $\left(x_{i-1}, x_{i+1}\right)$. Thus $\tau_{1}\left(x_{3}\right)=x_{2}, \tau_{3}\left(x_{2}\right)=x_{1}$ and $\tau_{2}\left(x_{1}\right)=x_{3}$. We shall define permutations $\tau_{i}^{\mathbf{x}}, 1 \leq i \leq 3$, on the set $Y=X \cup\left\{y_{1}, y_{2}, y_{3}\right\}$ assuming that none of $y_{i}$ belongs to $X$.

Set $\tau_{i}^{\mathbf{x}}\left(y_{i+1}\right)=y_{i-1}, \tau_{i}^{\mathbf{x}}\left(y_{i-1}\right)=y_{i+1}, \tau_{i}^{\mathbf{x}}\left(x_{i-1}\right)=y_{i}$ and $\tau_{i}^{\mathbf{x}}\left(y_{i}\right)=x_{i+1}$, and let $\tau_{i}^{\mathbf{X}}$ coincide with $\tau_{i}$ in all other cases, for all $i, 1 \leq i \leq 3$.

The cyclic structure of $\tau_{i}^{\mathbf{x}}$, for a given $i, 1 \leq i \leq 3$, differs from that of $\tau_{i}$ only slightly. There is a new cycle $\left(y_{i-1} y_{i+1}\right)$ of length two, and the cycle $\gamma_{i}=\left(\begin{array}{ll}\ldots x_{i-1} & \left.x_{i+1} \ldots\right)\end{array}\right)$ is changed into $\gamma_{i}^{\mathbf{x}}=\left(\ldots x_{i-1} y_{i} x_{i+1} \ldots\right)$. 
To see that $\tau_{1}^{\mathbf{x}} \tau_{2}^{\mathbf{x}} \tau_{3}^{\mathbf{x}}$ is the identity mapping it suffices to prove $\tau_{i+1}^{\mathbf{x}} \tau_{i-1}^{\mathbf{x}} \tau_{i}^{\mathbf{x}}(u)=u$ for all $u \in Y$ where $\tau_{i}^{\mathbf{x}}(u) \neq \tau_{i}(u)$ or where $\tau_{i}(u)$ is not defined. Now,

$$
\begin{array}{ll}
\tau_{i+1}^{\mathbf{x}} \tau_{i-1}^{\mathbf{x}} \tau_{i}^{\mathbf{x}}\left(x_{i-1}\right)=\tau_{i+1}^{\mathbf{x}}\left(y_{i+1}\right)=x_{i-1}, & \tau_{i+1}^{\mathbf{x}} \tau_{i-1}^{\mathbf{x}} \tau_{i}^{\mathbf{x}}\left(y_{i}\right)=\tau_{i+1}^{\mathbf{x}}\left(y_{i-1}\right)=y_{i} \\
\tau_{i+1}^{\mathbf{x}} \tau_{i-1}^{\mathbf{x}} \tau_{i}^{\mathbf{x}}\left(y_{i-1}\right)=\tau_{i+1}^{\mathbf{x}}\left(y_{i}\right)=y_{i-1}, \quad \text { and } & \tau_{i+1}^{\mathbf{x}} \tau_{i-1}^{\mathbf{x}} \tau_{i}^{\mathbf{x}}\left(y_{i+1}\right)=\tau_{i+1}^{\mathbf{x}}\left(x_{i}\right)=y_{i+1}
\end{array}
$$

We have verified that $\left(\tau_{1}^{\mathbf{x}}, \tau_{2}^{\mathbf{x}}, \tau_{3}^{\mathbf{x}}\right)$ fulfils conditions (P1)-(P2). Note that the number of both cycles and points increases by three. Hence the new surface, say $S^{\mathbf{x}}$, is connected of genus $g$ if and only if $S$ is connected of genus $g$. In particular, $S^{\mathbf{x}}$ is spherical when $S$ is spherical.

Let $\left(v_{1}, \ldots, v_{r}\right)$ be the vertices of a multigon $C$ in $S$. The cyclic sequence

$$
\left(\operatorname{sgn}_{C}\left(v_{1}\right), \ldots, \operatorname{sgn}_{C}\left(v_{k}\right)\right)
$$

will be called the signature of $C$. We know that if $\left(\varepsilon_{1}, \ldots, \varepsilon_{r}\right)$ is a signature of $C$, then $r \geq 3$ and $\sum \varepsilon_{j}$ is divisible by three, according to Lemma 2.1. The main purpose of this section is to show that for any such sequence $\left(\varepsilon_{1}, \ldots, \varepsilon_{r}\right)$ there exist a spherical surface $S$ and a triangle free multigon $C$ that yields $\left(\varepsilon_{1}, \ldots, \varepsilon_{r}\right)$ as its signature.

The next lemma shows that in our constructions one does not need to insist on triangle freeness.

Lemma 10.1. Let $C$ be a multigon of profile $\left(\sigma_{1}, \ldots, \sigma_{r}\right)$. Consider $j, 1 \leq j \leq r$, and assume that either $\left(\sigma_{j-1}, \sigma_{j}, \sigma_{j+1}\right)$ or $\left(\sigma_{j+1}, \sigma_{j}, \sigma_{j-1}\right)$ is a profile of a triangle, say $\mathbf{x}=x_{3} x_{2} x_{1}$. Then in $S^{\mathbf{x}}$ there exists a multigon $C^{\mathbf{x}}$ with profile that is obtained from the profile of $C$ by replacing all occurrences of $\sigma_{j-1}, \sigma_{j}$ and $\sigma_{j+1}$ by $\sigma_{j-1}^{\mathbf{x}}, \sigma_{j}^{\mathbf{x}}$ and $\sigma_{j+1}^{\mathbf{x}}$, respectively. The number of consecutive triples in the profile of $C^{\mathbf{x}}$ that induce a triangle in $S^{\mathrm{x}}$ is strictly smaller than the number of consecutive triples in the profile of $C$ that induce a triangle in $S$.

Proof. We can assume that $\sigma_{j}$ is a cycle of $\tau_{3}$ and that $\tau_{i}\left(x_{i-1}\right)=x_{i+1}, 1 \leq i \leq 3$. If $\left(\sigma_{j-1}, \sigma_{j}, \sigma_{j+1}\right)$ profiles a triangle, then $x_{3}, x_{2}$ and $x_{1}$ are consecutive vertices of $C$. Points $y_{1}, y_{3}$ and $y_{2}$ become points of the inner arcs of $\sigma_{j-1}^{\mathbf{x}}, \sigma_{j}^{\mathbf{x}}$ and $\sigma_{j+1}^{\mathbf{x}}$, respectively. The rest is easy.

Proposition 10.2. Let $\left(\varepsilon_{1}, \ldots, \varepsilon_{r}\right)$ be a cyclic sequence of integers 1 and -1 such that 3 divides $\varepsilon_{1}+\cdots+\varepsilon_{r}$, and $r \geq 3$. Then there exists a triangle free multigon $C$ in a spherical surface $S$ such that $\left(\varepsilon_{1}, \ldots, \varepsilon_{r}\right)$ coincides with the signature of $C$.

Proof. Thanks to Lemma 10.1 we can prove the statement without taking into consideration the requirement of triangle freeness. Suppose first that the solution is already known for all alternating sequences $(1,-1, \ldots, 1,-1)$.

To prove the rest we shall proceed by induction on $r$. Note that the solution for $\left(\varepsilon_{1}, \ldots, \varepsilon_{r}\right)$ yields a solution for $\left(-\varepsilon_{r}, \ldots,-\varepsilon_{1}\right)$, by changing to $C^{\text {op }}$. The cases $(1,1,1)$ and $(-1,-1,-1)$ are clear, as one can take any triple in a spherical surface $S$. (Of course, for that one has to know that at least one spherical surface exists. The number of examples is abundant, the smallest one is obtained from two distinct latin squares of order two.) 
Assume $r \geq 4$. We are assuming that $\left(\varepsilon_{1}, \ldots, \varepsilon_{r}\right)$ is not alternating, and so we can assume that $\varepsilon_{1}=\varepsilon_{2}=-1$. Let $C$ and $S$ be a solution to $\left(1, \varepsilon_{3}, \ldots, \varepsilon_{r}\right)$. Then there exist edges $\left(x_{3}, x_{2}\right)$ and $\left(x_{2}, x_{1}\right)$ of $C$ such that $x_{2}=v_{1}$ is the acute vertex. However that means that $\mathbf{x}=x_{3} x_{2} x_{1}$ is a triangle. Let us derive $C^{\mathbf{x}}$ from $C$ in such a way that the consecutive edges $\left(x_{3}, x_{2}\right)$ and $\left(x_{2}, x_{1}\right)$ are replaced by $\left(x_{3}, y_{1}\right),\left(y_{1}, y_{3}\right)$ and $\left(y_{3}, x_{1}\right)$. The signature of $C^{\mathbf{x}}$ is clearly equal to $\left(-1,-1, \varepsilon_{2}, \ldots, \varepsilon_{r}\right)$.

Note that $S^{\mathbf{x}}$ always contains a multigon with vertex sequence $\left(x_{3}, y_{1}, y_{3}, y_{2}\right)$, which yields the shortest alternating signature $(1,-1,1,-1)$. To show the existence of longer alternating sequences we need to expand one 1 to $1,-1,1$. Proceeding like above we replace consecutive edges $\left(x_{3}, x_{2}\right)$ and $\left(x_{2}, x_{1}\right)$ by $\left(x_{3}, y_{1}\right),\left(y_{1}, y_{2}\right),\left(y_{2}, y_{3}\right)$ and $\left(x_{3}, x_{1}\right)$.

\section{Main Theorem}

We are now ready to prove that any surface of positive genus that is derived from a separating latin bi-trade can be obtained by a flip-flop surgery from the surfaces of lower genus.

Lemma 11.1. Let $C$ and $D$ be triangle free multigons in the surface $S$ which is derived from $\left(\tau_{1}, \tau_{2}, \tau_{3}\right)$. Suppose that $C$ and $D$ are in different connected components of $S$. If $C$ is simple and $D$ separating, then every isogony of $C$ onto $D$ satisfies the conditions (H1)-(H3).

Proof. Conditions (H2) and (H3) are fulfilled trivially, as $C$ and $D$ are in different components. Condition (H1) simplifies to the requirement that an outer arc and an inner arc never have a common point, both in $C$ and $D$. For $C$ this is true because $C$ is simple, while for $D$ this follows from Lemma 3.3.

Call, like in the introduction, a combinatorial surface $S$ a trading surface if it can be obtained from a triple of permutations that satisfy condition (P1) and (P2).

Theorem 11.2. Let $S_{1}$ be a connected trading surface of genus $g \geq 1$. Then there exist a spherical trading surface $S_{2}$ and a connected trading surface $S$ of genus $g-1$ such that the disjoint union of $S_{1}$ and $S_{2}$ can be obtained by a flip-flop surgery in $S$ that maps a separating multigon onto a planar one.

Proof. The surface $S_{1}$ is not spherical, and hence it contains a non-separating simple triangle free multigon $C$ by Theorem 7.4. From Proposition 10.2 we know that there exists an isogonic multigon $D$ in some spherical trading surface $S_{2}$. Let $f$ be an isogony of $C$ onto $D$. By Lemma 11.1, the flip-flop surgery is possible. We obtain a surface $S$ which is connected, by Proposition 9.4 and Lemma 9.5. The reverse surgery thus yields the disjoint union of $S_{1}$ and $S_{2}$, by Proposition 8.3.

Let $s, s_{1}$ and $s_{2}$ be the sizes of $S, S_{1}$ and $S_{2}$, respectively. Furthermore, let $r, r_{1}$ and $r_{2}$ be their orders, and $g, g_{1}$ and $g_{2}$ their genera. We have $g_{2}=0, s=s_{1}+s_{2}$, $r=r_{1}+r_{2}, 2+s_{2}=r_{2}, 2+s_{1}=r_{1}+2 g_{1}$ and $2+s=r+2 g$, by Proposition 1.5. Hence $2 g=2+s_{1}+s_{2}-r_{1}-r_{2}=2\left(g_{1}-1\right)$. 
The multigon $D^{\prime}$ is planar by Proposition 9.1, and the multigon $C^{\prime}$ is separating, by Proposition 9.2.

Corollary 11.3. All trading surfaces can be obtained from the spherical ones by repeated applications of the flip-flop surgery.

Note that in the process of constructing a trading surface of positive genus one has to "throw away a ball" at every stage when the genus is increased. In other words, one has to forget about the component $S_{2}$ of Theorem 11.2. The efficiency of the process in some sense depends on the size of $S_{2}$, and should be considered in detail in the future.

\section{References}

[1] M. A. Armstrong, Basic topology. Springer 1983. MR705632 (84f:55001) Zbl 0514.55001

[2] N. Cavenagh, D. Donovan, A. Drápal, 3-homogeneous Latin trades. Discrete Math. 300 (2005), 57-70. MR2170114 (2006j:05027) Zbl 1073.05012

[3] N. Cavenagh, D. Donovan, A. Drápal, 4-homogeneous Latin trades. Australas. J. Combin. 32 (2005), 285-303. MR2139816 (2006e:05027) Zb1 1074.05020

[4] N. Cavenagh, P. Lisoněk, Planar Eulerian triangulations are equivalent to spherical Latin bitrades. J. Combin. Theory Ser. A 115 (2008), 193-197. MR2378864 (2008k:05029) Zbl 1131.05019

[5] N. J. Cavenagh, Embedding 3-homogeneous Latin trades into abelian 2-groups. Comment. Math. Univ. Carolin. 45 (2004), 191-212. MR2075269 (2005d:05028) Zbl 1099.05503

[6] N. J. Cavenagh, The theory and application of Latin bitrades: a survey. Math. Slovaca 58 (2008), 691-718. MR2453264 Zbl pre05382553

[7] A. Drápal, Latin Squares and Partial Groupoids (in Czech). Candidate of Science Thesis, Charles University, Prague, 1988.

[8] A. Drápal, Hamming distances of groups and quasi-groups. Discrete Math 235 (2001), 189197. MR1829848 (2002c:20037) Zbl 0986.20065

[9] A. Drápal, Geometry of Latin Trades. Manuscript circulated at the conference Loops'03, Prague, 2003.

[10] A. Drápal, On elementary moves that generate all spherical latin trades. Submitted.

[11] A. Drápal, T. Kepka, Exchangeable partial groupoids. I. Acta Univ. Carolin. Math. Phys. 24 (1983), 57-72. MR733686 (85f:08003) Zbl 0524.20048

[12] A. Drápal, T. Kepka, Exchangeable partial groupoids. II. Acta Univ. Carolin. Math. Phys. 26 (1985), 3-9. MR830261 (87e:08004) Zbl 0608.20051

[13] C. Hämäläinen, Partitioning 3-homogeneous Latin bitrades. Geom. Dedicata 133 (2008), 181193. MR2390076 (2009a:05030) Zbl pre05274035

[14] A. D. Keedwell, Critical sets for Latin squares, graphs and block designs: a survey. Congr. Numer. 113 (1996), 231-245. MR1393712 (97i:05017) Zbl 0955.05019

[15] A. D. Keedwell, Critical sets in latin squares and related matters: an update. Util. Math. 65 (2004), 97-131. MR2048415 (2005k:05047) Zbl 1053.05019 
[16] J. Lefevre, D. Donovan, N. Cavenagh, A. Drápal, Minimal and minimum size Latin bitrades of each genus. Comment. Math. Univ. Carolin. 48 (2007), 189-203. MR2338087 (2008f:05026)

Received 14 December, 2005; revised 8 May, 2008

A. Drápal, Deptartment of Mathematics, Charles University, Sokolovská 83, 18675 Praha 8, Czech Republic

Email: drapal@karlin.mff.cuni.cz 